\title{
Magnetic and Magnetoelectric Properties of Rare Earth Molybdates
}

\author{
B. K. Ponomarev ${ }^{1}$ and A. Zhukov ${ }^{2,3}$ \\ ${ }^{1}$ Institute of Solid State Physics, Russian Academy of Sciences, 142432 Chernogolovka, Russia \\ ${ }^{2}$ IKERBASQUE, Basque Foundation for Science, 48011 Bilbao, Spain \\ ${ }^{3}$ Departamento de Fisica de Materiales, Facultad de Quimicas, UPV/EHU, 20009 San Sebastian, Spain
}

Correspondence should be addressed to A. Zhukov, arkadi.joukov@ehu.es

Received 27 July 2011; Accepted 14 February 2012

Academic Editor: Mitsuteru Inoue

Copyright (C) 2012 B. K. Ponomarev and A. Zhukov. This is an open access article distributed under the Creative Commons Attribution License, which permits unrestricted use, distribution, and reproduction in any medium, provided the original work is properly cited.

\begin{abstract}
We present results on ferroelectric, magnetic, magneto-optical properties and magnetoelectric effect of rare earth molybdates (gadolinium molybdate, GMO, and terbium molybdate, TMO, and samarium molybdate, SMO), belonging to a new type of ferroelectrics predicted by Levanyuk and Sannikov. While cooling the tetragonal $\beta$-phase becomes unstable with respect to two degenerate modes of lattice vibrations. The $\beta-\beta^{\prime}$ transition is induced by this instability. The spontaneous polarization appears as a by-product of the lattice transformation. The electric order in TMO is of antiferroelectric type. Ferroelectric and ferroelastic GMO and TMO at room temperature are paramagnets. At low temperatures GMO and TMO are antiferromagnetic with the Neel temperatures $T_{\mathrm{N}}=0.3 \mathrm{~K}(\mathrm{GMO})$ and $T_{\mathrm{N}}=0.45 \mathrm{~K}(\mathrm{TMO})$. TMO shows the spontaneous destruction at $40 \mathrm{kOe}$ magnetic field. Temperature and field dependences of the magnetization in TMO are well described by the magnetism theory of singlets at $4.2 \mathrm{~K}$ $\leq T \leq 30 \mathrm{~K}$. The magnetoelectric effect in SMO, GMO and TMO, the anisotropy of magnetoelectric effect in TMO at $T=(1.8-$ 4.2) K, the Zeeman effect in TMO, the inversion of the electric polarization induced by the laser beam are discussed. The correlation between the magnetic moment of rare earth ion and the magnetoelectric effect value is predicted. The giant fluctuations of the acoustic resonance peak intensity near the Curie point are observed.
\end{abstract}

\section{Introduction}

At the end of 1960s, there was a great interest in the rare earth molybdates family $\mathrm{R}_{2}\left(\mathrm{MoO}_{4}\right)_{3}(\mathrm{RMO})(\mathrm{R}=$ $\mathrm{Pr}, \mathrm{Nd}, \mathrm{Sm}, \mathrm{Eu}, \mathrm{Gd}, \mathrm{Tb}$ and Dy) because these compounds exhibited the phenomena of ferroelectricity and ferroelasticity [1].

RMO from $\operatorname{Pr}_{2}\left(\mathrm{MoO}_{4}\right)_{3}$ (PMO) to TMO crystallizes to the tetragonal $\beta$-structure with a space group $\mathrm{P} \overline{4} 2 \mathrm{~m}$ (point symmetry group is $\overline{4} 2 \mathrm{~m}$ ). Their melting points are $1045^{\circ} \mathrm{C}$ for PMO and $1172^{\circ} \mathrm{C}$ for TMO. $\mathrm{Dy}_{2}\left(\mathrm{MoO}_{4}\right)_{3}$ (DMO) crystallizes to cubic $\gamma$-phase at $1222^{\circ} \mathrm{C}$ and transforms to $\beta$-structure at $1030^{\circ} \mathrm{C}$. While cooling the family undergoes a transformation from the tetragonal $\beta$-phase to the monoclinic $\alpha$-Phase. The temperatures of the $\beta-\alpha$ transformation are $987^{\circ} \mathrm{C}$ for $\mathrm{PMO}$ and $805^{\circ} \mathrm{C}$ for DMO.
However, the transformation is sluggish and, therefore, the high-temperature phase can be quenched in. If the thermodynamically metastable $\beta$-phases of RMO are further cooled, they undergo the second transformation leading to the lower symmetry ferroelastic-ferroelectric orthorhombic $\mathrm{Pba} 2 \beta^{\prime}$-structures (point symmetry group $\mathrm{mm} 2$ ). They are also thermodynamically metastable. The temperatures of $\beta$ $\beta^{\prime}$ phase transitions are $235^{\circ} \mathrm{C}$ for $\mathrm{PMO}$ and $145^{\circ} \mathrm{C}$ for DMO.

The single crystal samples of RMO are transparent in the visible light.

Both $\beta$ and $\beta^{\prime}$ phases are piezoelectric. The $\beta^{\prime}$ phase is ferroelectric. The spontaneous electric polarization values are of $\approx 2 \times 10^{-7} \mathrm{Coul} / \mathrm{cm}^{2}$. The dielectric permeability peak at the Curie temperature is of $\approx 10$ for GMO.

The ferroelastic properties are well exhibited. In a singledomain sample of (001) cut, the switching of the ferroelectric 
TABLE 1

\begin{tabular}{lcccc}
\hline The ion & The ground state & The spin, $S$ & $\begin{array}{c}\text { The angular } \\
\text { Momentum, } L\end{array}$ & $\begin{array}{c}\text { The total } \\
\text { Momentum, } J\end{array}$ \\
\hline $\mathrm{Sm}^{3+}$ & ${ }^{6} \mathrm{H}_{5 / 2}$ & $5 / 2$ & 5 & $5 / 2$ \\
$\mathrm{Gd}^{3+}$ & ${ }^{8} \mathrm{~S}_{7 / 2}$ & $7 / 2$ & 0 & $7 / 2$ \\
$\mathrm{~Tb}^{3+}$ & ${ }^{7} \mathrm{~F}_{6}$ & 3 & 3 & 6 \\
\hline
\end{tabular}

domain can be observed visually in polarized light at compressing the sample along the [010] axis.

Orthorhombic $\beta^{\prime}$-phases of rare earth molybdates are paramagnetic down to temperatures below $1 \mathrm{~K}$.

In $\mathrm{RMO}$ with $\mathrm{R}=\mathrm{Sm}, \mathrm{Gd}, \mathrm{Tb}$ and in mixed molybdates $\operatorname{DyGd}\left(\mathrm{MoO}_{4}\right)_{3}(\mathrm{DGMO}), \mathrm{TbGd}\left(\mathrm{MoO}_{4}\right)_{3}(\mathrm{TGMO})$ the nonlinear magnetoelectric effect was observed experimentally. In TMO and in TGMO, the switching of ferroelectric domains by the magnetic field was observed experimentally. These are the first substances in which the possibility was found to switch the ferroelectric domains by the magnetic field.

The longitudinal Zeeman effect was investigated in TMO in the magnetic field $H \leq 20 \mathrm{~T}$ along the [001] and [110] axes at $T=4.2 \mathrm{~K}$ and $1.7 \mathrm{~K}$. The experimental field dependences of the wave numbers of the absorption peaks $\nu_{i}(H)$ were obtained. The analysis of the dependences shows that the excited multiplet ${ }^{5} D_{4}$ of $\mathrm{Tb}^{3+}$ ion in the crystal field of TMO can be considered as consisting from five singlets and two quasidoublets. The field dependences of the energy levels $E_{i}(H)$ were obtained from the experimental data in the magnetic field along the [001] axis for the ${ }^{5} D_{4}$ multiplet. The experimental dependences $E_{i}(H)$ were described well by the theory of the magnetism of singlets.

The dependences of the photoinduced voltage in TMO upon the time $t$ of the illumination by a laser beam were measured at room temperature at $0.1 \mathrm{~s} \leq t \leq 4500 \mathrm{~s}$. The power of the laser radiation was $0.4 \mathrm{~W}$. The distribution of the intensity along the sample was varied from highly inhomogeneous to homogeneous. It was found that the photoinduced voltages of two types with the opposite signs appear due to the inhomogeneous illumination. The model was proposed to explain the appearance of the photoinduced voltage due to the inhomogeneous illumination.

The qualitative explanation of the mechanisms of these two effects was given. To make quantitative estimates, it is necessary to measure the dependences of the effect upon the temperature of the sample and upon the intensity of the light.

The temperature dependence of the intensity of the main acoustic resonant peak in GMO was measured at temperatures from $22^{\circ} \mathrm{C}$ to $165^{\circ} \mathrm{C}$. This temperature range includes the Curie point $\left(T_{\mathrm{C}}=159^{\circ} \mathrm{C}\right)$. The low-frequency fluctuations of the amplitude of the resonant current in GMO were observed near the Curie point. The corresponding variations of the measured voltage were 0.1 Volt. The typical times of the fluctuations were $(10-100) \mathrm{sec}$. The relative values of the corresponding fluctuations of the piezoacoustic impedance in GMO are two orders of magnitude larger than the values of the fluctuations of the physical parameters in the solids that were known before.
Such fluctuations of the physical properties of the solids were observed visually never before.

In [2], the point symmetry group $\mathrm{C}_{2}$ for the nearest surrounding of $\mathrm{Gd}^{3+}$ ions in GMO was established experimentally. The calculated value of the spontaneous polarization, assuming point charges $\mathrm{Gd}^{3+}$ and $\left(\mathrm{MoO}_{4}\right)^{2-}$, was $260 \cdot 10^{-9} \mathrm{Coul} / \mathrm{cm}^{2}$. This result can be considered as being in a satisfactory agreement with the experimental value $200 \cdot 10^{-9} \mathrm{Coul} / \mathrm{cm}^{2}$. All rare earth molybdates are isomorphic to GMO. So, the rare earth ions in RMO crystal lattice are assumed to be trivalent and the point symmetry group of the local crystal field at $\mathrm{R}^{3+}$ sites is assumed to be $\mathrm{C}_{2}$. The experimental data discussed below relates mostly to $\mathrm{Sm}_{2}\left(\mathrm{MoO}_{4}\right)_{3}, \mathrm{Gd}_{2}\left(\mathrm{MoO}_{4}\right)_{3}$, and $\mathrm{Tb}_{2}\left(\mathrm{MoO}_{4}\right)_{3}$. Table 1 contains the quantum numbers for corresponding free $\mathrm{R}^{3+}$ ions in the ground state.

It is worth mentioning that starting from 90-th magnetic shape-memory alloys (MSMAs) attract special interest owing to significant magnetic-field-induced strain (MFIS), also referred as the "magnetic shape-memory effect" originated from coupling between magnetic and structural ordering. Such strain arises through the magnetic-field-induced motion of twin boundaries [3]. Since the magnetic shapememory effect is useful for actuation purposes, the inverse effect may be utilized for sensing and energy harvesting applications [4]. Consequently, the direct and inverse magnetic shape-memory effects cause magnetic field-induced superelasticity, which is the magnetically induced recovery of a large mechanically induced deformation [4]. It is worth mentioning that, in fact, magnetic-field-induced strain (MFIS) effect has been previously observed and described in the same terms at the beginning of 90-th in ferroelectrics (rare-earth molybdates) [5]. Particularly, terbium molybdate shows so strong field-induced strain, that the spontaneous destruction in a constant magnetic field of $40 \mathrm{kOe}$ along the [100] axis at $T=1.5 \mathrm{~K}$ has been observed [5], but at that time, most attention has been paid to magnetic-fieldinduced electric polarization and magnetoelectric effects.

Latter family of magnetic shape memory alloys (MSMA) has been introduced, where the coupling between magnetic and structural ordering in conjunction with the magnetic and structural transformations giving rise to various functional properties: the magnetocaloric effect, the magneticfield-induced martensitic transformation, as well as its reverse transformation, giant magnetoresistance, and electric polarization [4].

In this paper, we will present the review on ferroelectric, magnetic, electric, and structural properties of the rare earth molybdates family $\mathrm{R}_{2}\left(\mathrm{MoO}_{4}\right)_{3}(\mathrm{RMO})(\mathrm{R}=$ $\mathrm{Pr}, \mathrm{Nd}, \mathrm{Sm}, \mathrm{Eu}, \mathrm{Gd}, \mathrm{Tb}$, and Dy). 


\section{Ferroelectric Properties}

Here, only a short description of ferroelectric properties of RMO will be given. The detailed review of this question is available in [1].

RMO belongs to a new type of ferroelectrics (improper ferroelectrics) theoretically predicted by Levanyuk and Sannikov [6].

While cooling the tetragonal $\beta$-phase becomes unstable with respect to two degenerate modes (soft modes) of lattice vibrations, this instability induces the displacive phase transition of the first order to the ferroelastic-ferroelectric $\beta^{\prime}$-GMO-type structure. The $\beta-\beta^{\prime}$ transition temperature is also the Curie temperature of the orthorhombic $\beta^{\prime}$-RMO. The spontaneous polarization appears at the transition as a by-product of the lattice transformation and cannot be considered as the order parameter. This conclusion was obtained for GMO by Petzelt and Dvořak [7] using group analysis. They confirmed this result experimentally by far-infrared reflectivity and transmission measurements in GMO.

Analogous result was obtained for TMO from neutronscattering experiments $[8,9]$. In [6], from the examination of the soft mode, it was shown that the primary-order parameter, which necessarily shows large fluctuations, near $T_{\mathrm{C}}$, have no macroscopic polarization but have instead antipolar. The freezing in of this antiferroelectric static displacement couples to the shear strain which in turn produces the electric polarization by piezoelectric coupling.

The displacive mechanism of $\beta-\beta^{\prime}$ transition is valid for all the members of RMO family because they are isomorphic to GMO and TMO.

The crystal structure of $\beta^{\prime}$-GMO was solved in $[2,10,11]$. Above the Curie temperature, the structure is tetragonal with space group $P \overline{4} 2_{1} m$ and point group $\overline{4} 2_{1} m$. Below the Curie temperature, it is orthorhombic with space group $\mathrm{Pba2}$ and point group $m m 2$. The reduction in point group symmetry from $\overline{4} 2_{1} m$ to $m m 2$ allows the formation of twin domains. They can be distinguished in polarized light. After cooling through the Curie point, the RMO single crystals always are in a polydomain state. When cooled through the Curie temperature, the equivalent [110] and [110] axes of the tetragonal phase become the [100] and [010] axes of the orthorhombic phase. These orthorhombic axes are unequal. The $b$ parameter along the [010] axis is larger than the $a$ parameter along the [100] axis: $(b-a) / a \approx 3 \times 10^{-3}$. So, the crystal changes its shape during the transition. The axes [100] and [010] of the tetragonal phase become the axes [11̄0] and [110] of the orthorhombic phase and the right angle between them changes by the value of $\approx 10^{\prime}$. This is the shear angle of the transition. It is determined by the relation $(b-a) / a$. The relative change of the [001] axis parameter $\Delta c / c$ during the transition is smaller than $10^{-4}$, but the structure of the [001] axis is changed drastically. It is not polar in the tetragonal phase and it becomes polar in the orthorhombic phase. This is the origin of the ferroelectric order.

The applied electric field along the [001] axis can reverse the [001] axis. This is accompanied by the mutual interchange of the orthorhombic [100] and [010] axes.
Due to the difference between $b$ and $a$ parameters, the mechanical compression along the [010] axis switches the orientational state. This is the origin of the ferroelasticity. A material is said to be ferroelastic if it has two or more stable orientational states in the absence of the external mechanical stress and if it can be reproducibly transformed from one to another of these states by the application of mechanical stress. Ferroelasticity was established in RMO by Aizu [12].

Ferroelasticity can be used to transform a multidomain sample into a single domain state. In a polydomain state due to a very small value of the shear angle, the direction of the [010] axes in domains with positive [001] axes almost coincides with the [100] axes in domains with negative [001] axes. So, a compression along [010] axes of positive domains makes them unstable. Simultaneously, this compression makes the negative domains more stable. Then, the domains with the [100] axes along the compression should grow and the domains with the [010] axes along the compression should disappear. This is the way to obtain a single domain sample.

The temperature dependence of the spontaneous electric polarization $P_{S}$ in GMO was measured in [13] over the temperature range from $4.2 \mathrm{~K}$ up to the Curie point $T_{\mathrm{C}}=$ $432.3 \mathrm{~K}$. The electric polarization takes values of $P_{\mathrm{S}} \approx 290 \times$ $10^{-9} \mathrm{Coul} / \mathrm{cm}^{2}$ at $T=4.2 \mathrm{~K}$ and $P_{\mathrm{S}} \approx 40 \times 10^{-9} \mathrm{Coul} / \mathrm{cm}^{2}$ at $T=T_{\mathrm{C}}=432.3 \mathrm{~K}$. The abrupt jump in the $P_{S}(T)$ curve was observed at $T=T_{\mathrm{C}}=432.3 \mathrm{~K}$ with a hysteresis loop of $\Delta T \approx 0.1 \mathrm{~K}$ width. This is a direct indication on the phase transition of the first order. Room temperature values of $P_{S}$ in SMO, EMO, GMO, and TMO are in the range of $P_{S} \approx$ $140 \times 10^{-9} \mathrm{Coul} / \mathrm{cm}^{2}$ for EMO and $P_{\mathrm{S}} \approx 240 \times 10^{-9} \mathrm{Coul} / \mathrm{cm}^{2}$ for SMO [14].

The temperature dependences of electric and elastic properties of GMO were measured in [15]. The dielectric permittivity of the clamped crystal $\varepsilon_{33}^{\kappa}$ does not depend on the temperature and shows no anomaly at the Curie point, while the elastic constant $c_{66}^{E}$ shows strong temperature dependence with a marked anomaly at the Curie point. The dielectric permittivity at constant and zero stress shows a weak anomaly at the Curie point due to the piezoelectric coupling. This result indicates that the ferroelectric ordering in GMO is a secondary effect. It appears as a consequence of the structural $\beta-\beta^{\prime}$ phase transition.

The pyroelectric properties of GMO were studied in [16, 17]. Near $T_{\mathrm{C}}$, hard-to-control spurious domain nucleation was observed. It was explained by small thermal gradients caused by the thermal signal being detected.

Both $\beta$ and $\beta^{\prime}$ phases of RMO are piezoelectric [17]. The electromechanical coupling coefficient is $4 \%$ at room temperature and $22 \%$ near $T_{\mathrm{C}}$.

The presence of the domain walls significantly influences the resonant behavior of a crystal.

\section{Magnetic Properties}

Magnetic properties of a single-crystal and single-domain spherical sample of GMO were investigated in [18-22]. In [18-20], the magnetization along the [001], [010] and [100] 
axes, respectively, was measured at temperatures from $0.3 \mathrm{~K}$ to $4.2 \mathrm{~K}$ in magnetic field up to $90 \mathrm{kOe}$. The saturation magnetization values at low temperatures and in high magnetic field along all crystal directions correspond to the magnetic moment $\mu_{S}=7 \mu_{B}$ which is typical for $\mathrm{Gd}^{3+}$ ion in the ground state ${ }^{8} S_{7 / 2}$. At temperatures above $\approx 1 \mathrm{~K}$, the field dependences of the magnetization show a typically paramagnetic behavior. At $T=0.5 \mathrm{~K}$, the field dependence of the magnetization exhibits a weak anomaly at $H=(2-$ 3) $\mathrm{kOe}$ that indicates on the field induced transformation in the antiferromagnetic structure from the antiparallel to parallel orientation of ionic magnetic moments [18]. At $T=$ $0.35 \mathrm{~K}$, this anomaly becomes more pronounced. On the basis of these data, the authors conclude that GMO becomes antiferromagnetic at temperatures lower than $\approx 0.3 \mathrm{~K}$.

Figure 1 shows the field dependences of the magnetization of GMO at $T=1 \mathrm{~K}$ in the magnetic field along the [001] axis (open circles), the [100] axis (triangles), and the [010] axis (crosses). These dependences were calculated from the tables published in [18-20]. The solid line is the Brillouin function $\mathrm{B}_{7 / 2}\left(\mu_{S} H / k T\right)$ that corresponds to a free $\mathrm{Gd}^{3+}$ ion in the ground state ${ }^{8} S_{7 / 2}$ as it should be in a usual paramagnetic substance. The Brillouin function describes qualitatively the experimental magnetization curve along the [001] axis. One can see that the magnetization of the paramagnetic GMO at $T=1 \mathrm{~K}$ is saturated at the magnetic field $H \approx 60 \mathrm{kOe}$. The

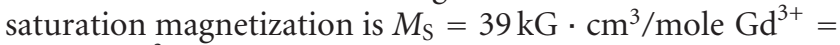
$6.98 \mu_{B} / \mathrm{Gd}^{3+}$ ion. This value agrees well with the magnetic moment $\mu_{\mathrm{S}}=7 \mu_{B}$ of $\mathrm{Gd}^{3+}$ ion in the ground state ${ }^{8} \mathrm{~S}_{7 / 2}$. The dependences of the magnetization in the magnetic field along the [100] and [010] axes are placed noticeably lower than for [001]. It is due to the natural magnetic crystalline anisotropy. The magnetization curves along the [100] and [010] axes are indistinguishable. It means that the [001] axis is the easy magnetization direction in GMO.

Figure 2 shows the experimental field dependence of the anisotropy energy for the magnetic field along the [100] axis. It was calculated from the data of Figure 1 using the relation:

$$
E_{a}(H)=\int_{0}^{H}\left[M_{[001]}(H)-M_{[100]}(H)\right] d H .
$$

In the state of the saturation $\left(H=H_{S} \approx 60 \mathrm{kOe}\right)$, the anisotropy energy is $E_{a}\left(H_{S}\right)=E_{S} \approx 10^{5} \mathrm{erg} / \mathrm{g} \approx 4.5 \times$ $10^{-5} \mathrm{eV} / \mathrm{Gd}^{3+}$ ion. This value is higher than the anisotropy constant of ferromagnetic iron at room temperature $\left(K_{1} \approx\right.$ $\left.0.6 \times 10^{5} \mathrm{erg} / \mathrm{g}\right)$. This is the energy needed to align all magnetic moments of $\mathrm{Gd}^{3+}$ ions in the paramagnetic GMO along the hard direction [100] at $T=1 \mathrm{~K}$.

Figure 3 shows the dependence of the anisotropy energy in GMO $E_{\mathrm{a}}\left(m_{\mathrm{r}}\right)$ upon the reduced magnetization $m_{r}=$ $M_{[100]} / M_{S}$ along [100]. $E_{\mathrm{a}}\left(m_{r}\right)$ is derived from

$$
E_{a}\left(M_{[100]}\right)=\int_{0}^{M_{[100]}}\left[H_{[100]}(M)-H_{[001]}(M)\right] d M
$$

by introducing a new variable $m_{r}=M_{[100]} / M_{S}$.

It is seen that this dependence is described well by the relation:

$$
E_{a}\left(m_{r}\right)=E_{S} m_{r}^{4}
$$

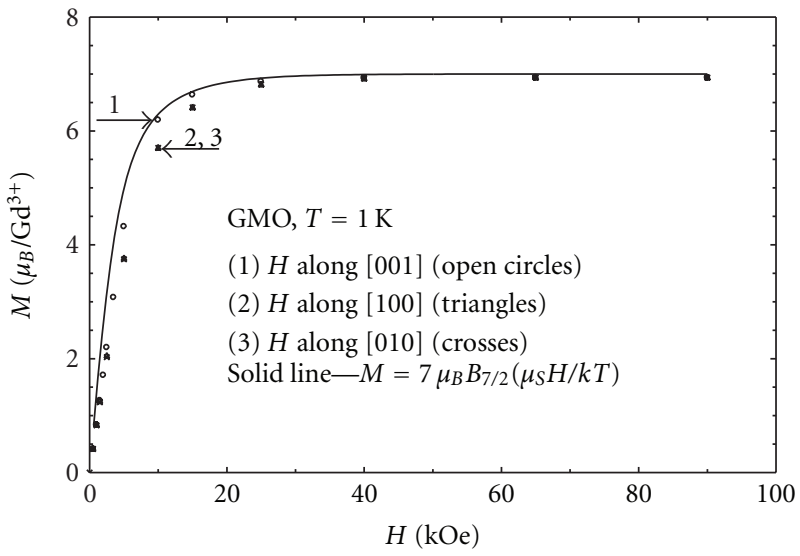

FIgure 1: The field dependences of the magnetization of GMO at $T=1 \mathrm{~K}$ in the magnetic field directed along the [001] axis (open circles), the [100] axis (triangles) and the [010] axis (crosses).

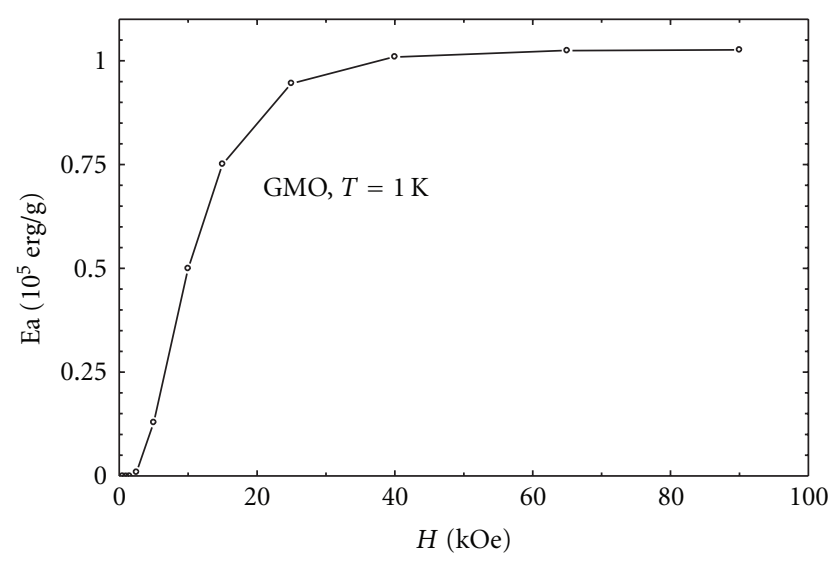

Figure 2: The field depends on the natural magnetic crystalline anisotropy energy upon the magnetic field along the [100] axis.

Here, $E_{S}$ is the anisotropy energy of a paramagnetic substance in a saturated state.

In [21], the magnetization curves of GMO were measured in the magnetic field along the [001] axis in the temperature range $(0.1-0.5) \mathrm{K}$ using the method of adiabatic demagnetizing. The sharp jump was observed on the isentropic magnetization curve in the magnetic field $H=$ $1.615 \mathrm{kOe}$. The temperature of the jump was $0.11 \mathrm{~K}$. The magnetic field $H=1.615 \mathrm{kOe}$ is the critical field of the destruction of the antiferromagnetic order in GMO at $T=$

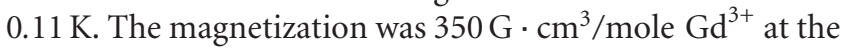

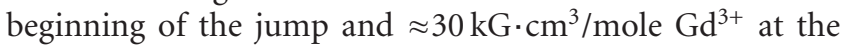
end of the jump. The magnetization curve between these two points was a vertical straight line. So, the phase transition from the antiferromagnetic to the paramagnetic state in GMO goes without the stage of the spin-flop.

The heat capacity of GMO was measured in the magnetic field up to $90 \mathrm{kOe}$ at the temperatures $(0.1-4.2) \mathrm{K}$ in papers [18-22]. The thermodynamic functions were calculated using the measured values of the magnetization and the heat capacity. 


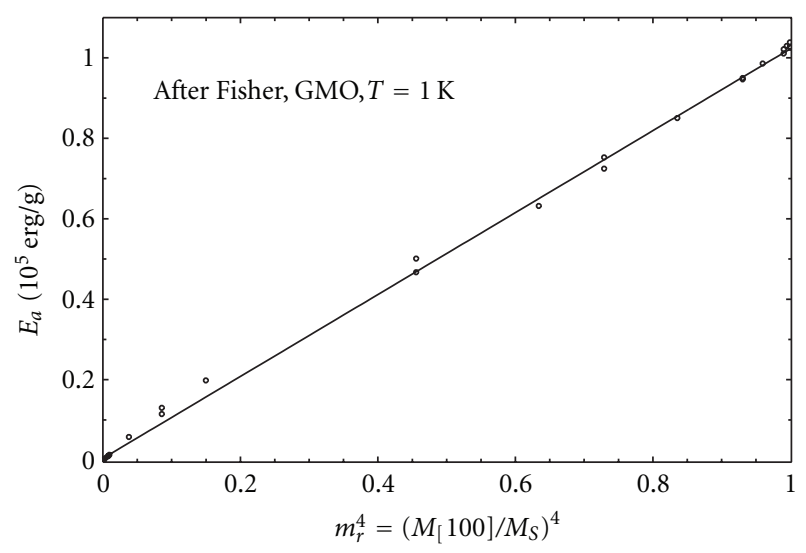

FIGURE 3: The energy of the natural magnetic crystalline anisotropy of GMO versus the reduced magnetization. The magnetic field is directed along the [100] axis.

In [23], the magnetization and heat capacity of a singlecrystal and single-domain spherical sample of TMO were measured over the range $(0.4-4.2) \mathrm{K}$ with fields up to $90 \mathrm{kOe}$ directed along the [001] axis. The magnetization was measured also along the [100] axis, but these measurements could not be performed in the field above $25 \mathrm{kOe}$ because of the mechanical destruction of the sample.

According to [23], the temperature dependences of the heat capacity at zero field and at $H=0.5 \mathrm{kOe}$ show $\lambda$ type anomalies with pronounced maxima at $T=0.44 \mathrm{~K}$ and $0.45 \mathrm{~K}$, respectively. As the magnetic field is raised, the $\lambda$ maxima shift toward the lower temperatures. The temperature dependences of the magnetization and magnetic susceptibility show the broad maxima around $T=1.6 \mathrm{~K}$. All of the above facts indicate on the antiferromagnetic ordering of the $\mathrm{Tb}^{3+}$ magnetic moments along the easy [001] axis with the Neel temperature $T_{\mathrm{N}} \approx(0.44-1.6) \mathrm{K}$.

Above these temperatures, the magnetization curves along the [001] axis look like for a paramagnetic substance at very low temperatures and in extremely high magnetic fields; but the experimental value of the magnetic saturation $M_{S}=7.7 \mu_{B} / \mathrm{Tb}^{3+}$ differs noticeably from $M_{S}=9 \mu_{B} / \mathrm{Tb}^{3+}$ for a usual paramagnetic substance with $\mathrm{Tb}^{3+}$ ion in the ground state ${ }^{7} \mathrm{~F}_{6}$. On the basis of this difference, the authors in [23] conclude that the total mechanical moment of the ground state doublet is $J_{z}= \pm 5$. In the following, we will show on the basis of our magnetic measurements and the observations of Zeeman effect that it is not correct. The unusual value of the magnetic moment in TMO can be explained strictly by the quantum mechanical theory of magnetism of singlets with nondiagonal matrix elements in the angular momentum operator of $\mathrm{Tb}^{3+}$ ion.

The magnetization curves along the [100] axis were measured only up to $25 \mathrm{kOe}$. The crystal was broken when the magnetic field was increased above $40 \mathrm{kOe}$. The authors explain the destruction of the sample by the magnetic torque. It is not a complete explanation of the self-destruction of TMO single crystal. Our later measurements showed that a sample of TMO at certain conditions can survive at $T=4.2 \mathrm{~K}$ even in the field of $200 \mathrm{kOe}$ along the [100] axis.
The absence of the magnetization data for TMO along the [100] axis at high magnetic fields makes it impossible to plot the anisotropy energy dependencies upon the magnetic field and the magnetization analogous to those for GMO on Figures 2 and 3. The estimate of the anisotropy energy of TMO at $H=25 \mathrm{kOe}$ and $T=1 \mathrm{~K}$ gives $E_{a} \approx 10^{6} \mathrm{erg} / \mathrm{g}$. From Figure 2, the corresponding value for GMO is $\approx 10^{5} \mathrm{erg} / \mathrm{g}$, that is, ten times lower than for TMO.

It was established from the measurements of the heat capacity in [23] that the relaxation time of the nuclear spin system of ${ }^{159} \mathrm{~Tb}^{3+}$ becomes very long at high fields and low temperatures. For example, in the magnetic field $H=90 \mathrm{kOe}$ at the temperature $T \approx 1 \mathrm{~K}$, the order of magnitude of the relaxation time is several hundred seconds. The heat capacity values taken below those temperatures are not in equilibrium. The higher the magnetic field the higher is, the temperature below which one cannot reach the equilibrium between the nuclear system and the lattice formed by electronic shells of ions. This phenomenon complicates the magnetic measurements in TMO at low temperatures and in high magnetic fields.

In [5], the magnetization of single-crystal single-domain samples of TMO was measured in pulsed magnetic fields up to $300 \mathrm{kOe}$ with pulse duration of $0.01 \mathrm{~s}$. The magnetization curves were measured at $T=4.2 \mathrm{~K}$ and $78 \mathrm{~K}$ along [001] axis and in the (001) plane. The magnetization process was adiabatic. The magnetization was highly anisotropic. The magnetization curves along the [001] axis were close to the paramagnetic saturation at both temperatures. At $T=78 \mathrm{~K}$, $H=300 \mathrm{kOe}$, the magnetization reached $7.2 \mu_{B} / \mathrm{Tb}^{3+}$ and at $T=4.2 \mathrm{~K}, H=240 \mathrm{kOe}$, it was $7.8 \mu_{B} / \mathrm{Tb}^{3+}$. Along the [010] axis, the magnetization was $3.4 \mu_{B} / \mathrm{Tb}^{3+}$ at $T=78 \mathrm{~K}$, $H=300 \mathrm{kOe}$, and $4.2 \mu_{B} / \mathrm{Tb}^{3+}$ at $T=4.2 \mathrm{~K}, H=240 \mathrm{kOe}$. The energy of the natural magnetic crystalline anisotropy of paramagnetic TMO was estimated qualitatively using the magnetization curves along the [001] axis and in the (001) plane. It proved to be of the order of $E_{a} \approx 2 \cdot 10^{7} \mathrm{erg} / \mathrm{g}$ at both temperatures. The magnetic anisotropy of the kind is unusual for a paramagnetic substance. It is the order of magnitude larger than the magnetic anisotropy constant of ferromagnetic cobalt $K_{1} \approx 10^{6} \mathrm{erg} / \mathrm{g}$ at $78 \mathrm{~K}$.

Figure 4 shows the magnetization curves of TMO in the plane $(001)$ at $T=4.2 \mathrm{~K}$. At the beginning of the pulse of the magnetic field, the [100] axis of the sample was oriented along the field. At $H=190 \mathrm{kOe}$, there was an anomaly on the magnetization curve. In the decreasing field, the magnetization curve was placed above that in the increasing field. Inspection of the sample in the polarized light after these measurements showed that the [100] and [010] axes were interchanged as compared to the state of the sample before switching on the pulse of the field. So, one could get the difference between the magnetization values for the field along the [100] and [010] axes. At $H=180 \mathrm{kOe}$, it was $\approx 0.6 \mu_{B} / \mathrm{Tb}^{3+}$. This interchange was observed also in $[24,25]$ from the measurements of the magnetostriction and the magnetoelectric effect. The interchange took place when the applied magnetic field was directed along the [100] axis and reached some critical value. In [26], the magnetization of 


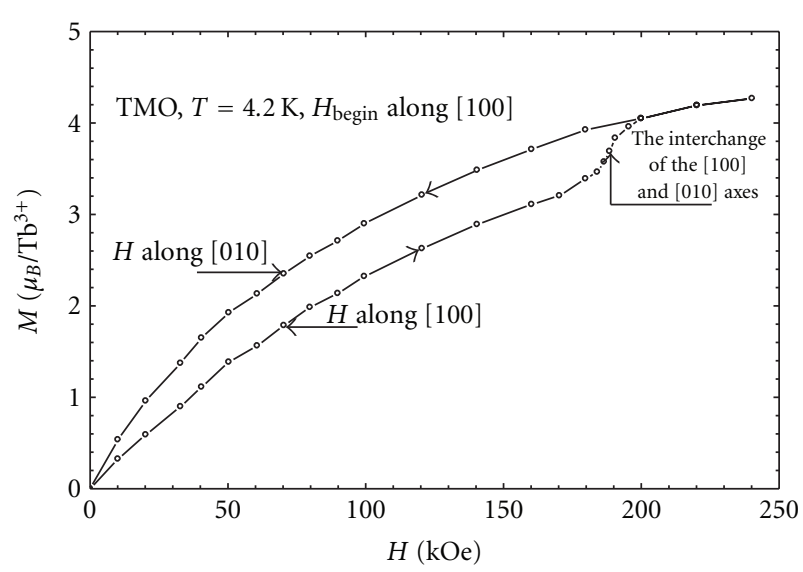

Figure 4: The magnetization isentropes in TMO at the initial temperature of the sample, $T=4.2 \mathrm{~K}$. On the lower branch, the magnetic field is directed along the [100] axis. The anomaly at $H \approx 190 \mathrm{kOe}$ shown by the arrow is due to the interchange of the [100] and [010] axes. On the upper branch, the field is parallel to the $[010]$ axis.

a single-crystal single-domain sample of TMO was measured in the direct current magnetic field up to $190 \mathrm{kOe}$ in the temperature range $T=(4.2-300) \mathrm{K}$. The magnetization curves at $T=4.2 \mathrm{~K}$ along the directions [001], [010] and [100] are shown on Figure 5. The magnetization curves along [010] and [100] on Figure 5 differ noticeably from the corresponding curves on Figure 4. The magnetization on Figure 4 is smaller than that on Figure 5. It is because the curves on Figure 5 are isotherms and those on Figure 4 are isentropes. The adiabatic process of magnetizing the sample on Figure 4 is accompanied by an increase of the temperature due to the magnetocaloric effect. According to our estimations, the temperature of the sample at $H=$ $240 \mathrm{kOe}$ on Figure 4 is $\approx(10-20) \mathrm{K}$ higher than at zero field. Respectively, the magnetization of Figure 4 is smaller than that of Figure 5.

There is no evidence of the interchange of the [100]↔ [010] axes of Figure 5. On the contrary, Figure 4 shows the obvious transition of the sample from the state with the [100] axis parallel to the field into the state with the [010] axis parallel to the field at $H=190 \mathrm{kOe}$. This can be explained by the temperature dependence of the critical field of the [100]↔[010] interchange $H_{\mathrm{cr}}(T)$ and the magnetocaloric effect in pulsed fields. Our measurements of the magnetoelectric effect showed that the critical field $H_{\text {cr }}(T)$ decreases from approximately $130 \mathrm{kOe}$ at $T=$ $10 \mathrm{~K}$ down to $30 \mathrm{kOe}$ at $T=30 \mathrm{~K}$. The $[100] \leftrightarrow[010]$ interchange of Figure 4 was observed because of increasing the temperature due to the magnetocaloric effect and the corresponding decreasing of $H_{\mathrm{cr}}(T)$.

It is seen that, along the easy direction [001], the saturation magnetization per one terbium ion $M_{S} \approx 8 \mu_{B} / \mathrm{Tb}^{3+}$ is not equal to the magnetic moment of a free $\mathrm{Tb}^{3+}$ ion $M_{\mathrm{Tb}^{3+}}=9 \mu_{B} / \mathrm{Tb}^{3+}$. This is the result of the interaction of the angular momentum of $\mathrm{Tb}^{3+}$ ion with the crystal field. So, to describe the experiment, one should use the singlet magnetism theory.
Before describing this theory, it is very useful to remind the main starting points of the standard theory of paramagnetism of noninteracting ions.

The Zeeman energy is given by the following equation:

$$
\widehat{V}_{Z}=-\hat{\mu}_{L} \vec{H}-\hat{\mu}_{S} \vec{H}=-\mu_{B}(\widehat{L}+2 \hat{S}) \vec{H}=-\mu_{B} g_{J} \widehat{J H} .
$$

The Hamiltonian of the problem is

$$
\hat{H}=\hat{H}_{\mathrm{CR}}+\hat{V}_{Z}=H_{\mathrm{CR}}-g_{J} \mu_{B}\left(H_{x} \hat{J}_{x}+H_{y} \hat{J}_{y}+H_{z} \hat{J}_{z}\right) ;
$$

$\hat{H}_{\mathrm{CR}}$ is the Hamiltonian of the crystal field; $\hat{J_{x}}, \hat{J_{y}}$, and $\hat{J}_{z}$ are operators of projections of the total mechanical momentum on the $x, y$, and $z$ axes. This consideration gives only one of three contributions to the magnetic moment of the ion-the paramagnetism of orientation. We do not consider here the paramagnetism of polarization according to Van Vleck and the diamagnetism of precession.

Consider a free $\mathrm{Tb}^{3+}$ ion in the magnetic field along $z$ axis. This is the case of the full degeneracy of the orbital multiplet:

$$
\begin{gathered}
\hat{H}_{\mathrm{CR}}=0 ; \\
H_{x}=H_{y}=0 .
\end{gathered}
$$

From (5) and (6), the Hamiltonian of a free ion is obtained:

$$
\begin{gathered}
\hat{H}=-g_{J} \mu_{B} H_{z} \hat{J}_{z} ; \\
\hat{J_{z}}=-i \frac{\partial}{\partial \varphi} .
\end{gathered}
$$

Here, $\varphi$ is the angle in the $x, y$ plane. The $\varphi$-dependent parts of normalized wave functions of $\hat{J}_{z}$ for degenerate levels of the multiplet of a free ion are [27]

$$
|J, m\rangle=\Phi_{m}(\varphi)=\frac{\exp (i m \varphi)}{\sqrt{2 \pi}} .
$$

The fact is that a wave function is given by only one exponent plays a decisive role.

Due to this fact, only diagonal elements of the Hamiltonian (7) can have non zero values. So, the secular equation is

$$
\prod_{m=J}^{-J}\left(g \mu_{B} m H_{z}-E_{m}\right)=0 .
$$

Solutions of (10) give the eigenvalues of the energy of a free ion:

$$
\begin{gathered}
E_{m}=g \mu_{B} m H_{z} ; \\
m=-J,(-J+1), \ldots, 0, \ldots,(J-1), J .
\end{gathered}
$$

The components of the degenerate multiplet (11) possess the field independent magnetic moments:

$$
M_{z m}=-\frac{\partial E_{m}}{\partial H_{z}}=-g \mu_{B} m
$$




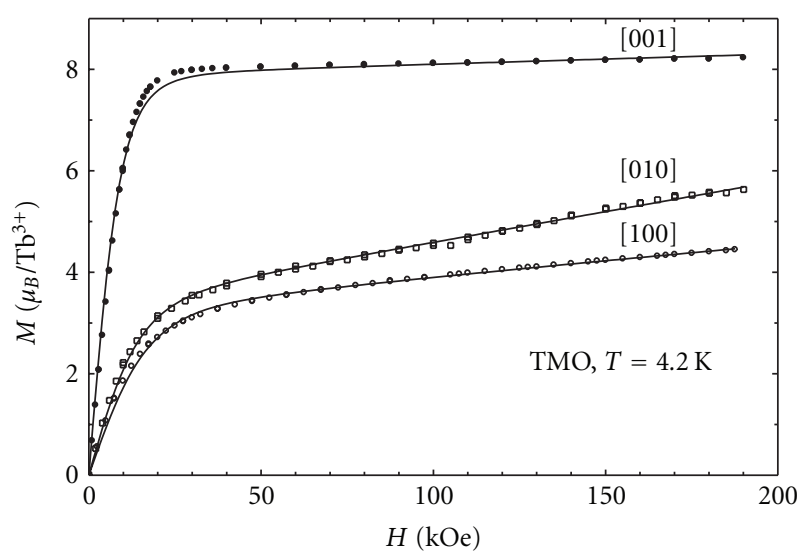

Figure 5: The magnetization curves of TMO at $T=4.2 \mathrm{~K}$ along main crystallographic directions. Solid lines are calculated using the theory of magnetism of singlets.

Using (11) and (12) and the statistics of Maxwell-Boltzman, one obtains the Brillouin function to describe the field and temperature dependence of the magnetization in a system of free ions:

$$
\begin{aligned}
M_{z}(H, T)= & g J \mu_{B} B_{J}\left(\frac{g J \mu_{B} H_{z}}{k T}\right) \\
= & g J \mu_{B}\left[\frac{2 J+1}{2 J} \operatorname{cth}\left(\frac{2 J+1}{2 J} \times \frac{g J \mu_{B} H_{z}}{k T}\right)\right. \\
& \left.\quad-\frac{1}{2 J} \operatorname{cth}\left(\frac{1}{2 J} \times \frac{g J \mu_{B} H_{z}}{k T}\right)\right] .
\end{aligned}
$$

The total mechanical momentum of $\mathrm{Tb}^{3+}$ ion in ${ }^{7} \mathrm{~F}_{6}$ ground state is

$$
J=L+S=3+3=6
$$

$L$ and $S$ are the angular and spin moments, respectively. The $g$-factor is [28]

$$
g=1+\frac{J(J+1)+S(S+1)-L(L+1)}{2 J(J+1)}=\frac{3}{2} .
$$

The corresponding saturation magnetic moment is $\mu_{S}=$ $g J \mu_{B}=9 \mu_{B}$.

The singlet magnetism theory describes the magnetic properties of ions in the crystal field. It is based on the Jahn-Teller theorem [29]. According to this theorem, the surrounding of the ion cannot be stable if the ground state of the ion in the crystal field is degenerate (excluding the case of Kramers degeneracy for ions with an odd number of outer electrons [30]). The spontaneous rearrangement of the crystal lattice goes on because the development of the instability reduces the symmetry of the crystal field and removes the degeneracy of the ground state. It means that rare earth ions with the even number of $4 \mathrm{f}$ electrons unavoidably have the singlet ground state [31]. If two lowest singlets are separated by a narrow gap of the order of $2 \Delta_{0} \approx(1-5) \mathrm{cm}^{-1}$ and are much more away from other singlets, then the former two singlets can be considered as a quasidoublet. The magnetic moment of the ion with the quasidoublet as a ground state should be calculated by the singlet magnetism theory [31].

The main distinction of this theory from the standard theory of the paramagnetism of free ions (6)-(16) is that the wave functions of the orbital singlets cannot be described by only one exponent like (9). The crystal field splits the degenerate multiplet of a free ion that is given by (11)(12) and mixes the wave functions (9) of these levels. The wave functions of the singlets are linear combinations of the functions given by (9) with the values of $m$ given by (12). The forms of the combinations are determined by the symmetry of the crystal field and of the operators: $\hat{J}_{x}, \hat{J}_{y}$, and $\hat{J}_{z}$.

The point symmetry group of the surrounding of $\mathrm{Tb}^{3+}$ ion in TMO is $\mathrm{C}_{2}$ [2]. For this symmetry, the Hamiltonian matrix for the magnetic field along $z$ axis is

$$
\left|\begin{array}{cc}
-\Delta_{0} & g J_{\mathrm{eff}} \mu_{B} H_{z} \\
g J_{\mathrm{eff}} \mu_{B} H_{z} & +\Delta_{0}
\end{array}\right|
$$

Here, $2 \Delta_{0}$ is the splitting of energy levels of the quasi-doublet under consideration.

$J_{\text {eff }}$ is a nondiagonal matrix element of the total mechanical momentum operator. It is given by the relation:

$$
J_{\text {eff }}=\left\langle\psi_{z}\left|\hat{J_{x}}\right| \psi_{S}\right\rangle=6 a_{6}^{S} a_{6}^{z}+4 a_{4}^{S} a_{4}^{z}+2 a_{2}^{S} a_{2}^{z} ;
$$

$\psi_{S}$ and $\psi_{z}$ are the wave functions of the lower and upper levels of the quasidoublet, respectively. The difference between $M_{S}$ and $M_{\mathrm{Tb}^{3+}}$ is not too large. This result indicates that the main harmonics that form the quasidoublet are $(|6,6\rangle-$ $|6,-6\rangle) / \sqrt{2}$ and $(|6,6\rangle+|6,-6\rangle) / \sqrt{2}$. From here, it follows

$$
\begin{aligned}
& \psi_{\mathrm{S}}=\frac{1}{\sqrt{2}}\left[\mathrm{a}_{6}^{\mathrm{S}}(|6,6\rangle+|6,-6\rangle)\right. \\
&\left.+\mathrm{a}_{4}^{\mathrm{S}}(|6,4\rangle+|6,-4\rangle)+\mathrm{a}_{2}^{\mathrm{S}}(|6,2\rangle+|6,-2\rangle)+\mathrm{a}_{0}^{\mathrm{S}}\right] ; \\
& \psi_{\mathrm{z}}=\frac{1}{\sqrt{2}}\left[\mathrm{a}_{6}^{\mathrm{z}}(|6,6\rangle-|6,-6\rangle)\right. \\
&\left.\quad+\mathrm{a}_{4}^{\mathrm{z}}(|6,4\rangle-|6,-4\rangle)+\mathrm{a}_{2}^{\mathrm{z}}(|6,2\rangle-|6,-2\rangle)\right] .
\end{aligned}
$$

One can see from (17) that for an ion with the ground state in the form of a quasidoublet the diagonal matrix elements do not depend upon the magnetic field and the nondiagonal elements are not zero and depend upon the magnetic field. This is a main distinction of the magnetic properties of the spectrum of singlets compared to the degenerate spectrum (11). The secular equation of the Hamiltonian (17) is

$$
\left|\begin{array}{ll}
-\Delta_{0}-E & g J_{\mathrm{eff}} \mu_{B} H_{z} \\
g J_{\mathrm{eff}} \mu_{B} H_{z} & +\Delta_{0}-E
\end{array}\right|=0 .
$$

From (20), one obtains the eigenvalues of the energy of the quasi-doublet $E_{1,2}$ in the magnetic field $H_{z}$ :

$$
E_{1,2}=\mp \sqrt{\Delta_{0}^{2}+\left(g J_{\text {eff }} \mu_{B} H_{z}\right)^{2}} .
$$


Here, $E_{1}$ - the energy of the lower level of the quasidoublet; $E_{2}$ - the energy of the upper level of the quasidoublet. Magnetic moments of the components of the quasidoublet are given by the following relation:

$$
M_{z 1,2}=-\frac{\partial E_{1,2}}{\partial H_{z}}= \pm \frac{2 g^{2} J_{\mathrm{eff}}^{2} \mu_{B}^{2} H_{z}}{2 \sqrt{\Delta_{0}^{2}+\left(g J_{\mathrm{eff}} \mu_{B} H_{z}\right)^{2}}} .
$$

There is a principal distinction between ions with the nondegenerate spectrum (21) and ions with the degenerate spectrum (11). The energy levels of ions with the degenerate spectrum (11) have the field independent magnetic moments (13). These moments are not zero in the absence of the magnetic field if $m \neq 0$.

As it is seen from (22), the magnetic moments of the levels of the quasidoublet essentially depend upon the magnetic field. They are zero at zero magnetic field. In other words, the ion with the nondegenerate spectrum of singlets can be magnetically polarized in the magnetic field. This is the paramagnetism of the polarization. So we came to the recognition of a very important fact that the idea of some definite and field independent magnetic moment of the ion is applicable only to a narrow class of problems in which the potential of the ion has the spherical symmetry and the spectrum of the ion is degenerate. The sample of the kind is GMO where $\mathrm{Gd}^{3+}$ ion in the ground state ${ }^{8} \mathrm{~S}_{7 / 2}$ has zero angular momentum and hence the spherical symmetry of the ionic potential. The overwhelming majority of problems, and TMO among them, insist on taking account of the field dependence of the ionic magnetic moment. This is done in the singlet magnetism theory by the relation (22).

Using the Maxwell-Boltzman statistics, one can obtain the field and temperature dependence of the magnetization per ion for a singlet magnetic substance in the field directed along the [001] axis:

$$
M_{z}(H, T)=\frac{g^{2} J_{\mathrm{eff}}^{2} \mu_{B}^{2} H_{z}}{\sqrt{\Delta_{0}^{2}+\left(g J_{\mathrm{eff}} \mu_{B} H_{z}\right)^{2}}} \cdot \operatorname{th} \frac{\sqrt{\Delta_{0}^{2}+\left(g J_{\mathrm{eff}} \mu_{B} H_{z}\right)^{2}}}{k T} .
$$

This is a two-level version of the singlet magnetism theory. It does not describe the anisotropy of the experimental results shown in Figure 5. It gives a zero value for the magnetization along the [100] and [010] axes. To describe the anisotropy, one should take account of not less than three lowest singlets of the spectrum.

The nearest surrounding of $\mathrm{Tb}^{3+}$ ion in $\mathrm{TMO}$ is an octahedron formed by seven oxygen ions [2]. The point symmetry group of the octahedron can be described as $\mathrm{C}_{2}$. The axis of the second order is a polar axis of the octahedron. Due to this symmetry, the [100] and [010] components of the magnetic moment $M_{x}$ and $M_{y}$ are zero in the two singlet model. Nonzero values for $M_{x}$ and $M_{y}$ appear when taking account of the nearest excited singlet. This is a three-level model of singlet magnetism theory.

The sites of $\mathrm{Tb}^{3+}$ ions in TMO are of two types. The directions of the polar axes of the octahedrons at nonequivalent sites are approximately opposite to each other and parallel or antiparallel (approximately) to the [001] axis. The octahedrons at the nonequivalent sites are rotated with respect to each other by the angle of approximately $90^{\circ}$ around the [001] axis. It means that the axes of the easy magnetization in the (001) plane should be different for ions disposed at the nonequivalent sites: the angle between them should be of $90^{\circ}$.

Taking account of the nonequivalent sites for $\mathrm{Tb}^{3+}$ ions, one obtains in the three-level modification of the theory the following expression for the field and the temperature dependence of the magnetization:

$$
\begin{aligned}
M_{p}\left(H_{p}, T\right)=\sum_{i=1,2} & \frac{\mu_{i p}^{2} H_{p} \operatorname{th}\left(B_{i p} / k T\right) / B_{i p}}{2\left(1+\exp \left(d_{i p} / k T\right) / 2 \operatorname{ch}\left(B_{i p} / k T\right)\right)} \\
& +\chi_{p} H_{p} .
\end{aligned}
$$

Here, $p$ indicates the direction in the crystal and takes values [001], [010] and [100].

$i$ indicates the type of the site. $B_{i p}=\sqrt{b_{i p}^{2}+\left(\mu_{i p} H_{p}\right)^{2}}$. $H_{p}$ is a projection of the field on the direction $p . M_{p}$ is a projection of the magnetic moment per one $\mathrm{Tb}^{3+}$ ion on the direction of the magnetic field. $\mu_{i p}$ are the saturation magnetic moments of $\mathrm{Tb}^{3+}$ ions at sites of two types for main directions in the crystal. $\chi_{p}$-Van Vleck susceptibility along the direction $p$. $k$-Boltzmann constant. $b_{i p}$ and $d_{i p}$ are coefficients of the theory that can be found from energy gaps $\Delta_{n}$ and $\delta_{m}$ between the singlets. $\Delta_{n}$ is the energy gap between the $n$-th excited singlet and the ground singlet for the sites of the first type, $\delta_{m}$ is the same for the sites of the second type. $\Delta_{n}$ and $\delta_{m}$ can be taken from the photoluminescence spectrum of $\mathrm{Tb}^{3+}$ ion in TMO [5]: $\Delta_{1} \approx \delta_{1}=(2.7 \pm$ $0.35) \mathrm{cm}^{-1} ; \Delta_{2} \approx \delta_{2}=(5.4 \pm 0.35) \mathrm{cm}^{-1}$. Using these values of $\Delta_{n}$ and $\delta_{m}$, the coefficients $b_{i p}$ and $d_{i p}$ were found. Then, the magnetization curves $M_{p}\left(H_{p}\right)$ were calculated at the temperature $T=4.2 \mathrm{~K}$ for the directions [001], [010] and [001] by fitting expression (24) to the corresponding experimental curves. In these calculations, the coefficients $\mu_{i p}$ and $\chi_{p}$ were used as the fitting parameters. It was found that the values $\mu_{1[001]}=\mu_{2[001]}=7.9 \mu_{B} / \mathrm{Tb}^{3+}$ along the [001] axis are identical for the nonequivalent sites. Along the [100] axis, the saturation magnetic moments for nonequivalent sites are different: $\mu_{1[100]}=6.4 \mu_{B} / \mathrm{Tb}^{3+} \gg \mu_{2[100]}=1 \mu_{B} / \mathrm{Tb}^{3+}$. For the [010] direction, the opposite nonequality occurs: $\mu_{1[010]}=0.05 \mu_{B} / \mathrm{Tb}^{3+} \ll \mu_{2[010]}=6.9 \mu_{B} / \mathrm{Tb}^{3+}$. This result reflects the difference in the symmetry of the nonequivalent sites of $\mathrm{Tb}^{3+}$ ions. A good agreement with the experiment takes place at $T=4.2 \mathrm{~K}$ for main directions. One can see that the difference between the experimental saturation magnetic moment of TMO and the magnetic moment of free $\mathrm{Tb}^{3+}$ ion in the ground state ${ }^{7} \mathrm{~F}_{6}$ is explained completely by the singlet magnetism theory. So, the additional assumption about the ground state of $\mathrm{Tb}^{+}$ion in TMO with $J= \pm 5[23]$ is not necessary.

The comparison of the magnetization curves of GMO and TMO indicates definitely the role of the angular momentum $L$ of the trivalent rare earth ion in the formation of the magnetic properties in rare earth molybdates. 
The $L$ value determines the main contribution to the energy $E_{a}$ of the natural magnetic crystalline anisotropy. In GMO, $\mathrm{Gd}^{3+}$ ion in the ground state ${ }^{8} \mathrm{~S}_{7 / 2}$ has $L=0$, and the energy of anisotropy in the magnetically saturated state is $E_{a} \approx 10^{5} \mathrm{erg} / \mathrm{g}$. The magnetization reaches the saturation along hard directions [100] and [010] at $H \approx 60 \mathrm{kOe}$ (see Figures 1 and 2). In $\mathrm{TMO}, \mathrm{Tb}^{3+}$ ion in the ground state ${ }^{7} \mathrm{~F}_{6}$ has $L=3$, and the magnetization along hard directions [100] and [010] is far from saturation even in the magnetic field $H=190 \mathrm{kOe}$ (see Figure 5). The energies of anisotropy at $H=190 \mathrm{kOe}$ found using relation (1) are $E_{a[100]} \approx 1.13 \cdot 10^{7} \mathrm{ergg}$ and $E_{a[010]} \approx 0.96 \cdot 10^{7} \mathrm{ergg}$ that is two orders of magnitude larger than in GMO. There is a strong correlation between the angular momentum value and the anisotropy energy. The ion with the nonzero angular momentum has a nonspherical charge cloud of the outer $4 \mathrm{f}$ electrons. Magnetizing the crystal containing nonspherical ions like $\mathrm{Tb}^{3+}$ rotates these ions and hence changes the coupling between the ions and the crystal field much more than in the crystal with spherically isotropic ions like $\mathrm{Gd}^{3+}$. So, TMO is magnetically more anisotropic than GMO. The anisotropy energy values for TMO found in [26] agree by the order of magnitude with the value $E_{a} \approx 2 \cdot 10^{7} \mathrm{erg} / \mathrm{g}$ found from the measurements in pulsed fields in [5]. The difference is mainly due to the adiabatic character of magnetizing in [5] in contradiction to the isothermal one in [26].

The samples investigated in $[5,26]$ were not destroyed in contradiction to the result of [23] despite that magnetic fields in $[5,27]$ were three times higher than in [23]. It means that the magnetic torque acting on the sample in $[5,26]$ was also larger than in [23]. So, the reason of the destroying the sample in [23] was not the magnetic torque in despite of conclusion made in [23].

The rotation of the nonspherical rare earth ions while magnetizing RMO crystals distorts the crystal lattice of RMO. The distortions of a ferroelectric crystal lattice unavoidably change its electric polarization due to the piezoelectric effect. This is the origin of the magnetoelectric effect to be discussed in the following section.

\section{Magnetoelectric Effect}

The presence of rare earth ions with nonzero angular momentum and nonzero total momentum in the crystal lattice of a ferroelectric substance leads to the large magnetoelectric effect (MEE). Rare earth ions with nonzero angular momentum possess the spatially anisotropic charge cloud of outer 4 f-electrons. The spatial anisotropy of the magnetic ions determines large values of the natural magnetic crystalline anisotropy and the magnetostriction. These properties in the ferroelectric crystal ensure the strong dependence of the electric polarization upon the magnetic field, that is, MEE.

MEE was predicted in [32], but the consideration of the problem was restricted by the linear magnetoelectric effect. It was established that this effect is possible only in the antiferromagnetic crystals. In [33], the prediction of linear MEE was given for a specific substance $\mathrm{Cr}_{2} \mathrm{O}_{3}$. The first experimental observations of linear MEE in $\mathrm{Cr}_{2} \mathrm{O}_{3}$ were done in $[34,35]$.

The most important point of MEE in rare earth molybdates is the absence of the restrictions related to the magnetic structure. The general consideration of the problem shows that nonlinear MEE in rare earth molybdates can exist in the paramagnetic state.

The first observation of nonlinear MEE in the paramagnetic tetragonal $\mathrm{NiSO}_{4} \cdot 6 \mathrm{H}_{2} \mathrm{O}$ was published in [36].

In [37], nonlinear MEE was observed in paramagnetic TMO at $T=77 \mathrm{~K}$. That was the first observation of MEE in the rare earth molybdate family. In [38], MEE was measured in TMO and GMO in pulsed magnetic fields up to $110 \mathrm{kOe}$ at $T=(77-290) \mathrm{K}$.

Figure 6 shows the field dependence of the magnetically induced electric polarization (MEP) in TMO at $T=77 \mathrm{~K}$ [38]. The field was applied along the orthorhombic axis [1]0]. MEP was measured along the [110] axis. As the spontaneous polarization $\bar{P}_{S}$ is directed along the [001] axis, this result can be considered as the rotation of the $\bar{P}_{S}$ in the magnetic field.

The dependence of MEP upon the magnetic field is quadratic with a good accuracy. $\mathrm{MEP}$ is $P=4.12$. $10^{-9} \mathrm{Coul} / \mathrm{cm}^{2}$ at $H=10 \mathrm{~T}$. It is small compared to the spontaneous electric polarization $P_{S}=180 \cdot 10^{-9} \mathrm{Coul} / \mathrm{cm}^{2}$ [14].

MEP in GMO was equal to zero in the limit of the experimental error.

The standard symmetry consideration [39] shows that, at a given geometry of the experiment, the components of the magnetically induced electrical polarization in the orthorhombic paramagnetic crystal are proportional to the products of the magnetization components:

$$
\begin{aligned}
& P_{x} \sim M_{x} \cdot M_{z} \\
& P_{y} \sim M_{y} \cdot M_{z} .
\end{aligned}
$$

For a usual paramagnetic crystal,

$$
M_{z}=\chi_{z z} H_{z}
$$

Here $x, y, z$ correspond to the [100], [010], [001] axes, respectively; $\chi_{z z}$ is a component of the diagonal tensor of the magnetic susceptibility. It follows from (25) and (26) that the effect on Figure 6 should be zero.

The contradiction between the result of calculations [39] and the experimental observation of the nonzero effect in $[37,38]$ can be eliminated by taking account of the local symmetry of $\mathrm{Tb}^{3+}$ ions surrounding. As it was noted in Section 3, the nearest surrounding of $\mathrm{Tb}^{3+}$ ion in TMO is an octahedron formed by seven oxygen ions [2]. The octahedron has the $\mathrm{C}_{2}$ point symmetry group. The axis of the second order is a polar axis of the octahedron. The symmetry axes of the octahedron do not coincide with the symmetry axes of the crystal. The polar axis of the octahedron makes an angle with the [001] axis, and the magnetic field applied in the (001) plane has a nonzero projection on the polar axis of the octahedron. According to the theory of magnetism of singlets, the magnetic field 


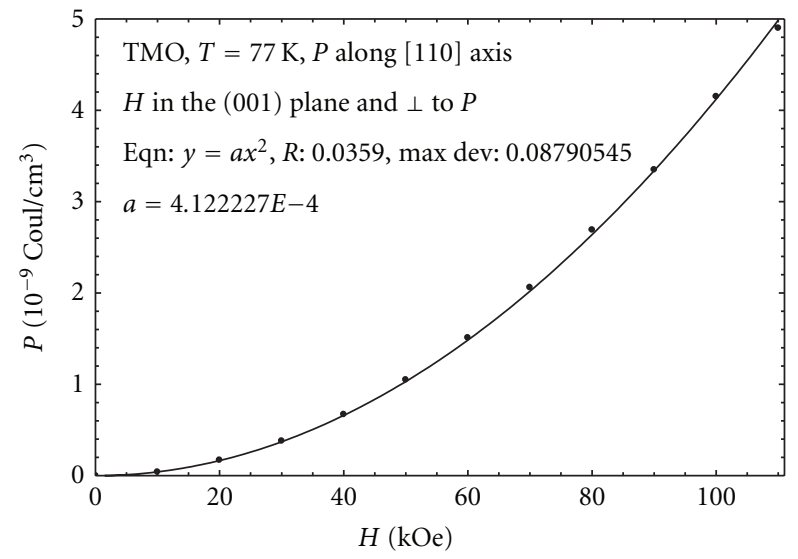

Figure 6: The field dependence of the magnetically induced electric polarization in TMO along the [110] axis at $T=77 \mathrm{~K}$. The pulsed magnetic field is directed along the $[1 \overline{1} 0]$ axis. The solid line is the least-square fitting by $P=p_{0} H^{2} \cdot p_{0}=4.12 \cdot 10^{-19} \mathrm{Coul} \cdot \mathrm{cm}^{-2} \cdot \mathrm{Oe}^{-2}$.

applied along the polar axis of the octahedron magnetically polarizes $\mathrm{Tb}^{3+}$ ion with the magnetic moment along the polar axis. So, the magnetic field applied in the (001) plane of the crystal induces a magnetic moment on $\mathrm{Tb}^{3+}$ ion along the polar axis of the octahedron. This moment, in turn, has a nonzero projection on the [001] axis of the crystal and, consequently, induces the electric polarization components along [100] and [010] axes according to (25). Hence, the bulk magnetic susceptibility tensor of TMO single crystal should have nonzero nondiagonal components and (26) should be replaced by

$$
M_{z}=\left(\chi_{x z}+\chi_{y z}+\chi_{z z}\right) H_{z} .
$$

Then, there would be no contradiction between the measurements [37, 38] and the calculations [39]. The relation (27) should be verified experimentally.

The magnetic susceptibility tensor of an individual $\mathrm{Tb}^{3+}$ ion with the main directions along the symmetry axes of the oxygen octahedron has only diagonal components.

At the beginning of this section, it was pointed out on the role of the angular momentum of a rare earth ion. A rare earth ion with a nonzero angular momentum has a nonspherical charge cloud of magnetoactive 4 f-electrons. This charge cloud is coupled strongly with an ionic magnetic moment due to a strong spin-orbit interaction. It means that the rotation of the ionic magnetic moment by means of the magnetic field should result in the rotation of the charge cloud. The rotation of a nonspherical charge cloud induces strains in a crystal lattice-the magnetostriction. This should change the electric polarization of a crystal due to the piezo-effect giving rise to MEE. The effect is large in those molybdates, in which rare earth ions have large magnetic moments $\mu_{S}=g J \mu_{B}$ and nonzero angular moments $L$.

In line with this mechanism, it was suggested that the MEP was due to the change of the spontaneous electric polarization of TMO induced by the single ion magnetostriction of $\mathrm{Tb}^{3+}$ ions [38]. This suggestion is the base of the magnetostriction model of MEE in rare earth molybdates. According to this model, the dependence of MEP upon the inverse temperature squared should be linear at high temperatures and at not very strong field. This can be shown as follows. MEP is caused by the piezoe-ffect. So it depends linearly on the strains. The strains are due to the magnetostriction. The magnetostriction at high temperatures and at not very strong magnetic field is proportional to the magnetization squared. The magnetization at these conditions is inversely proportional to the temperature. This leads to the linear dependence of MEP upon the inverse temperature squared $P\left(T^{-2}\right)$. This prediction was confirmed experimentally in [38].

Figure 7 shows the experimental dependence $P\left(T^{-2}\right)$ in TMO. MEP was measured along the [110] axis in pulsed magnetic field of $104.5 \mathrm{kOe}$ directed along the [1힐 axis. Solid line presents the least-square fitting. One can see that the prediction is fulfilled well.

The movement of the ferroelectric domain walls initiated in $\mathrm{TbGd}\left(\mathrm{MoO}_{4}\right)_{3}$ (TGMO) by the pulsed magnetic field of $100 \mathrm{kOe}$ at $T=78 \mathrm{~K}$ was observed in $[40,41]$. It was the first observation of the reversal of the spontaneous electric polarization due to the magnetic field.

Figure 8 shows the field dependence of the electric polarization in a single-domain sample of TMO at $T=$ $20 \mathrm{~K}$ [42]. The polarization was measured along the [001] axis that is along the spontaneous electric polarization. In the initial state, the magnetic field was directed along the axis [100]. The negative sign was ascribed to the initial value of the spontaneous electric polarization on Figure 8. It is seen from Figure 8 that the magnetic field directed along the [100] axis decreases the absolute value of the spontaneous polarization. This decreasing is due to the negative paraprocess in the ferroelectric subsystem that is the decreasing of the parameter of the ferroelectric order. The negative paraprocess continues up to a critical field $H=H_{C}$ at which the magnetoelastic strains become large enough to nucleate a new domain. The new domain differs from the old one by the opposite direction of $\bar{P}_{S}$ and by the mutual interchange of [100] and [010] axes. In orthorhombic rare earth molybdates, the ferroelectric domains are identical to the structural twin domains.

The samples investigated in [42] were put into the single domain state by the mechanical compression along the [010] axis. In the samples of this kind, the nucleation of the reversed domain usually goes on by arising of a flat domain wall in the (110) or (1 $\overline{10})$ plane. This is the beginning of the reversal of electric polarization. The reversal results in a jump of the electric polarization near a critical value of $H=H_{C} \approx 50 \mathrm{kOe}$.

The process of the displacement of the domain wall starts when the new domain reaches the critical size and begins to grow. In Figure 8, the start of the displacement takes place at $H \approx 45 \mathrm{kOe}$. The new domain grows at the expense of the old one. During the growth, the domain wall move in the direction normal to its plane that is along the [110] or

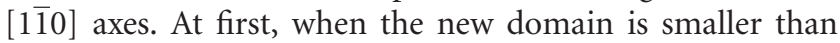
the old one, the absolute value of polarization of the sample being the sum of opposite polarizations of the two domains 


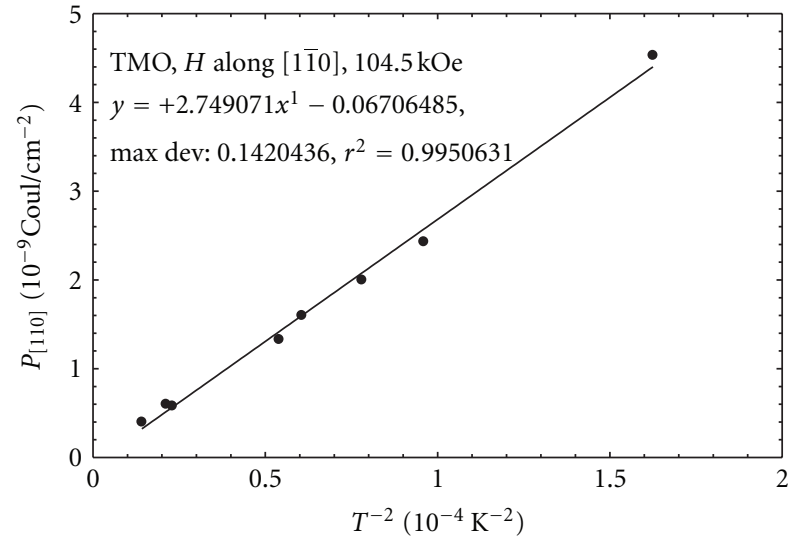

FIGURE 7: The dependence of the electrical polarization in TMO along the [110] axis in pulsed magnetic field of $104.5 \mathrm{kOe}$ directed

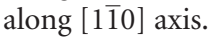

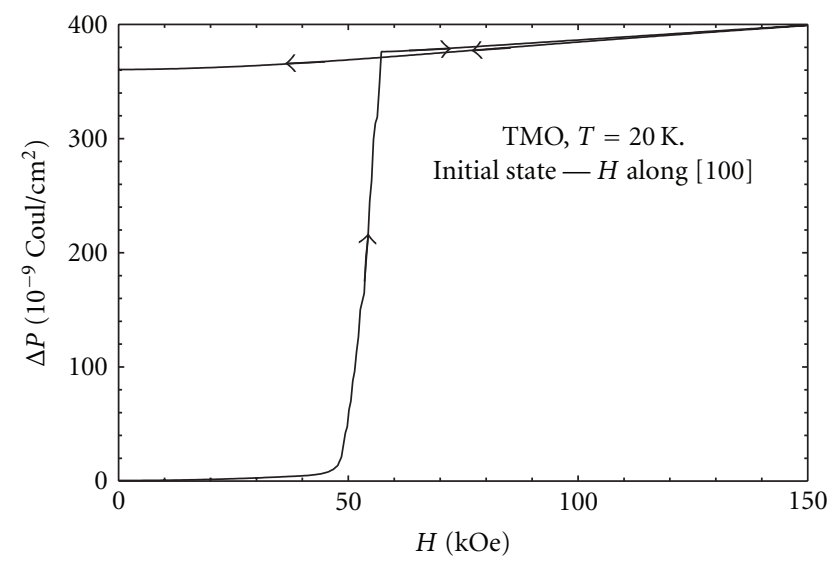

Figure 8: The field dependence of the electric polarization in TMO measured along the [001] axis. $T=20 \mathrm{~K}$. At $H<50 \mathrm{kOe}$, the field was directed along the [100] axis. At $H>50 \mathrm{kOe}$, the field was directed along the [010] axis.

is decreasing. At $H \approx 53 \mathrm{kOe}$, the opposite domains are equal in size $(P=0)$. Further, the absolute value of the polarization began to grow. If the critical field is applied for a long time, the new domain occupies all the volume of the sample. At $H \approx 57 \mathrm{kOe}$, the reversal of the electric polarization is completed and the displacement process ceases.

After this, the increase of the polarization goes on by the mechanism of the positive paraprocess.

The examination of the sample in polarized light after the measurements of Figure 8 showed that after the jump the axes [100] and [010] were mutually interchanged. It is known that this interchange is accompanied by the reversal of the electric polarization. This supports the above interpretation of Figure 8.

These experiments were the first to realize the reversal of the electric polarization in a ferroelectric crystal without using the electric field. The temperature dependence $H_{C}(T)$ was measured in [42]. While decreasing the temperature from $100 \mathrm{~K}$ down to $30 \mathrm{~K}$, the critical field decreases from
$60 \mathrm{kOe}$ to $30 \mathrm{kOe}$. Further decrease of the temperature results in an increase of the critical field up to $130 \mathrm{kOe}$ at $T=15 \mathrm{~K}$. At lower temperatures down to $4.2 \mathrm{~K}$, the reversal of the electric polarization was not observed up to $200 \mathrm{kOe}$.

During the growth of the new domain in TMO, small rotations of the tetrahedrons $\mathrm{MoO}_{4}$ take place [2]. These rotations create defects in the crystal lattice. The more defects are in the lattice the larger is the critical field. That is why every reversal of the spontaneous electric polarization increases the critical field. When the number of the reversal cycles was large enough the sample was mechanically destroyed into small particles at the next in turn reversal.

Figure 9 shows the field dependence of MEP with a sharp break down of MEP at $H=71.5 \mathrm{kOe}$. It is due to the mechanical destruction of the sample. Before the destruction, the spontaneous polarization of the sample was reversed 5 times at temperatures from $98 \mathrm{~K}$ to $20 \mathrm{~K}$. One can compare this number of complete reversals with the only attempt to magnetize the sample of TMO along the [100] axis in Fishers experiment [23]. That attempt failed at $H=40 \mathrm{kOe}$ because of the destruction of the sample. To the authors' opinion, the reasons of the destruction were the spherical shape and the pin holes of Fishers sample. The pin holes were the origins of a large number of the domain walls of different orientations. The domain walls intersected each other and very large stresses were concentrated at those intersections. These stresses destroyed the sample. The spherical shape stopped the movement of the domain walls because when a flat domain wall moves through the sphere, the area of the wall has to change its size and this also generates the stresses with the evident destruction. The samples used in [42] were rectangular parallelepipeds. They had dimensions $7 \times 7 \times 1 \mathrm{~mm}^{3}$. The large face of the sample was the (001) plane and the small faces were the (110) and ( $1 \overline{1} 0)$ faces. At this orientation, the domain walls were always parallel to the small faces and normal to the large face of the sample. This orientation ensured the easiest conditions for the domain walls to move. So, the samples sustained several reversals of the spontaneous electric polarization and several tens of magnetizing up to $H=200 \mathrm{kOe}$ along the [100] axis without the reversal.

The anisotropy of MEE in TMO was investigated experimentally in [43] in the magnetic field up to $60 \mathrm{kOe}$ at temperatures $4.2 \mathrm{~K} ; 1.8 \mathrm{~K}$ and at $T=0.39 \mathrm{~K}$ up to $H=$ $100 \mathrm{kOe}$. MEP was measured along the [001] axis. The magnetic field was applied in the (001) plane. The angle $\varphi$ between the [010] axis and the magnetic field was varied from 0 to $90^{\circ}$. The experimental angular dependences of MEP did not obey the relations of the standard theory of the anisotropy of MEE in a paramagnetic crystal derived in [36]. According to [36], the dependence of MEP in a tetragonal paramagnetic crystal upon the strength of the magnetic field and upon its direction can be described by the relation:

$$
\Delta P=\xi H^{2} \cdot \sin 2 \varphi
$$

Here, $\varphi$ is the angle between the magnetic field and the tetragonal axis; $\xi$ is the constant of the theory. 


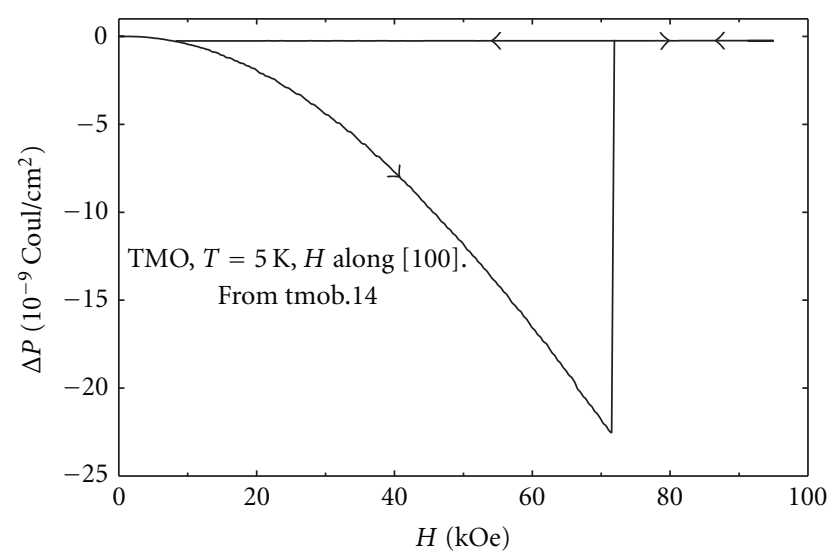

Figure 9: The field dependence of MEP in TMO at $T=5 \mathrm{~K}$. The field is directed along the [100] axis. The positive direction of the spontaneous polarization coincides with that one of the $\Delta P$ axis. A sharp breakdown of MEP at $H=71.5 \mathrm{kOe}$ is due to the mechanical destruction of the sample.

Figure 10 displays some of the experimental isotherms of MEP measured along the [001] axis in TMO at $4.2 \mathrm{~K}$ (curves $1-5)$ and at $0.39 \mathrm{~K}$ (curves $6-10)$ for various directions of the field in the (001) plane. The sign of $\Delta P(H)$ in Figure 10 was determined by comparing it with the sign of the jump of the electric polarization when the spontaneous polarization switches in the critical magnetic field (see Figure 8). The field dependences of MEP are nonlinear. MEP is positive in the magnetic field directed along the [010] axis (a positive paraelectric process in the ferroelectric subsystem at $\varphi=$ 0 , curves 1 and 6). MEP is negative in the magnetic field directed along the [100] axis (a negative paraprocess at $\varphi=$ $90^{\circ}$, curves 5 and 10 ).

The field dependences $\Delta P(H)$ do not agree with (28) and what is more unusual is that the $\Delta P(H)$ itself depends upon the direction of the field.

The angular dependences of MEP in TMO do not agree with (28) neither.

Figure 11 displays some of the experimental angular dependences of MEP in TMO at temperatures $T=4.2 \mathrm{~K}$ (curves $1-3$ ) and $T=0.39 \mathrm{~K}$ (curve 4 ). Curves 1, 2, 3 were measured at $H=20 \mathrm{kOe}, 40 \mathrm{kOe}$, and $60 \mathrm{kOe}$, respectively. Curve 4 was measured at $H=100 \mathrm{kOe}$. The line 4 is drawn for the eyes guide. The lines 1-3 are calculated following the theory of mean quadrupole moments $[43,44]$. The points refer to the experimental values of $\Delta P(H, \varphi)$. Their arrangement is not symmetric relative to $\varphi=45^{\circ}$ contrary to (28). The absolute values of $\Delta P(\varphi=0)$ and $\Delta P\left(\varphi=90^{\circ}\right)$ are different. The values of $\Delta P\left(\varphi=45^{\circ}\right) \neq 0$. So, (28) of the theory [36] does not describe MEE in TMO. Obviously, a new approach is needed to describe MEE in TMO. This approach was developed in [44] and applied to the problem in [43] using the experimental field dependences of the magnetization [26].

The magnetic-field-induced electric polarization in crystals (MEP) is, like the magnetostriction and magnetic linear birefringence, an even-parity magnetic effect. The classical

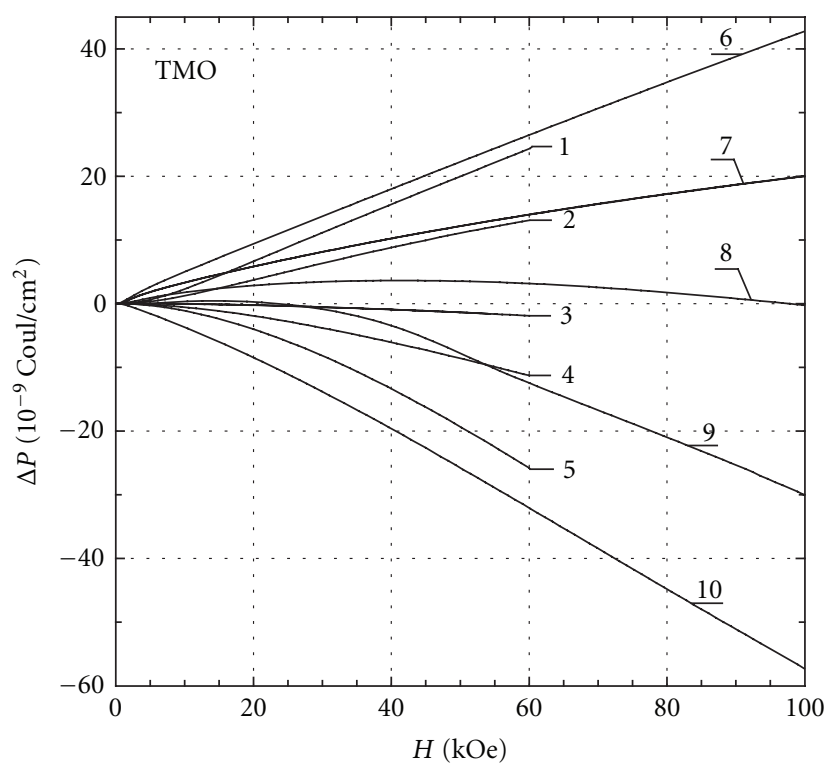

FIgURE 10: MEP in TMO induced along the [001] axis by the magnetic field applied in the (001) plane for various values of the angle $\varphi$ between the field and the [010] axis at $T=4.2 \mathrm{~K}$ (curves $1-5$ ) and $T=0.39 \mathrm{~K}$ (curves $6-10)$; (1) $\varphi=0^{\circ}$; (2) $35.5^{\circ}$; (3) $57.7^{\circ}$; (4) $66.6^{\circ}$; (5) $90^{\circ}$; (6) $0^{\circ}$; (7) 35.5 ; (8) $57.7^{\circ}$; (9) $68.8^{\circ}$; (10) $90^{\circ}$.

theory of even effects was developed in $[45,46]$. This theory describes well the properties of weakly anisotropic compounds, in particular, of those containing d-ions. The situation with f-ions is more complicated. According to [44], even magnetic effects in rare earth compounds are governed by magnetic susceptibilities of the mean multipole moments of the rare earth ions 4f-shell. Their calculation requires knowledge of the energy levels and wave functions of these ions. It was established in [26] that the ground state of $\mathrm{Tb}^{3+}$ ions in TMO is apparently a combination of three close-lying singlets, which belongs to different irreducible representations of the symmetry group of the ion environment and are separated by $2.7 \mathrm{~cm}^{-1}$ in energy.

In this case, at the magnetic field in the (001) plane, the following relation takes place [43]:

$$
\begin{aligned}
\Delta P_{z}(T, H, \varphi)= & A H_{x} M_{x}(T)+B H_{y} M_{y}(T)+C H_{x} M_{y}(T) \\
& +D H_{y} M_{x}(T)+E H_{x}^{2}+F H_{x} H_{y}+G H_{y}^{2} .
\end{aligned}
$$

Here, $T$ is the temperature. It should not be very high, but the condition $T \gg T_{N}$ should be fulfilled. $T_{N}$ is the temperature of the antiferromagnetic ordering. In TMO, $T_{N}=0.45 \mathrm{~K}$ [23].

$A, B, C, D, E, F$, and $G$ are constants of the theory which do not depend on the temperature; $H_{x}=H_{[010]}$ is the magnetic field component along the [010] axis:

$$
M_{x}=M_{[010]}-\chi_{V V[010]} H_{[010]} \text {; }
$$

$M_{[010]}$ is the magnetization component along the [010] axis. 
$H_{y}=H_{[100]}$ is the magnetic field component along the [100] axis:

$$
M_{y}=M_{[100]}-\chi_{V V[100]} H_{[100]}
$$

$M_{[100]}$ is the magnetization component along the [100] axis.

$\chi_{V V[010]}$ and $\chi_{V V[100]}$ are the Van Vleck susceptibilities along the [010] and [100] axes, respectively. $M_{x}$ and $M_{y}$ are the magnetization components of the $\mathrm{Tb}^{3+}$ ion quasitriplet in the (001) plane. The values of the constants $A, B, C, D, E, F$, and $G$ were determined in [43] using corresponding experimental dependences $\Delta P(H)$ from [43] and $M_{x, y}\left(H_{x, y}\right)$ from [26]. The solid lines 1,2 , and 3 on Figure 11 were calculated using (29), the values of $A, B, C, D, E, F, G$, and the dependences (30) and (31) that were calculated in turn for various values of $\varphi$ from the experimental data of [26]. It is seen that the theory agrees well with the experiment. A satisfactory agreement was obtained in analogous calculations for $T=$ $1.8 \mathrm{~K}$. At $T=0.39 \mathrm{~K}$, there is no agreement between the theory and the experiment because the theory does not take account of the antiferromagnetic order that arises at $T_{N}=$ $0.45 \mathrm{~K}$.

Four mechanisms of changing the electric polarization were established in TMO: the positive and negative paraelectric processes, the rotation of the electric polarization, and the displacement of the domain wall.

As it was noted above, MEE in $\mathrm{Gd}_{2}\left(\mathrm{MoO}_{4}\right)_{3}(\mathrm{GMO})$ at $T=77 \mathrm{~K}$ was not observed. This fact is in a qualitative agreement with the magnetostrictive model [38]. However, MEE in GMO is not forbidden utterly by this model. In spite of a zero angular momentum of $\mathrm{Gd}^{3+}$ ion, the natural magnetic crystalline anisotropy in GMO is not zero. The calculations based on data of Figure 1 derived from results of [18-20] give for the anisotropy energy of GMO in the magnetically saturated state at $T=1 \mathrm{~K}$ the value of $\approx 10^{5} \mathrm{erg} / \mathrm{g}$. It is higher than the anisotropy constant in iron at room temperature. It is well known that the existence of the magnetic crystalline anisotropy involves the existence of the magnetostriction. From these reasoning, it follows that MEE can exist in GMO at low temperatures. MEP in GMO was measured in [47] along the [001] axis at $T=(5-300) \mathrm{K}$ for two directions of the magnetic field along the [100] and [010] axes and for $H \leq 190 \mathrm{kOe}$.

At $T=5 \mathrm{~K}$ and $H=190 \mathrm{kOe}, \mathrm{MEP}$ was $\Delta P \approx$ $0.3 \cdot 10^{-9} \mathrm{Coul} / \mathrm{cm}^{2}$. This fact confirmed the magnetostrictive model of MEE. In [48], the anisotropy of MEE in GMO was investigated in the magnetic field up to $200 \mathrm{kOe}$ at temperatures $T=4.2 \mathrm{~K}$ and $0.4 \mathrm{~K}$. The experimental angular dependences of MEP at $T=0.4 \mathrm{~K}$ and various field values are shown on Figure 12. The solid lines are calculated by (28) shifted along the $\varphi$ axis by $\varphi_{0}=\pi / 2$. One can see that the experimental dependences $\Delta P(\varphi)$ agree with the relation (28) through the whole field range investigated. The experimental dependences $\Delta P(\varphi)$ at $T=4.2 \mathrm{~K}$ agree with (28) as well. It is evident that at higher temperatures, the theory [36] developed for a paramagnetic substance should work also. So, (28) of the standard theory [36] describes the angular dependences of MEE in GMO satisfactorily in

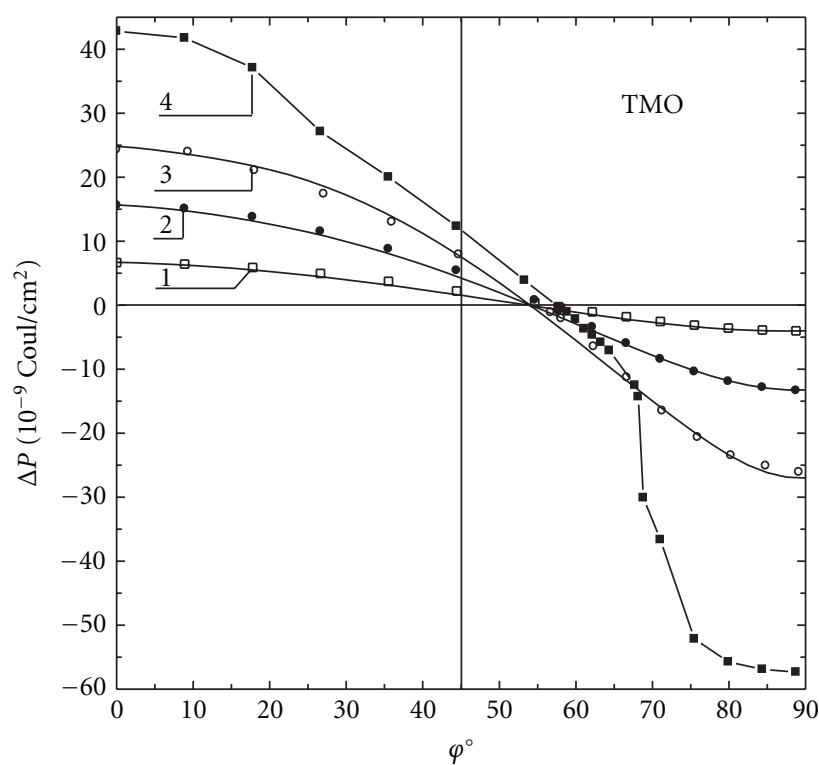

FIgure 11: The angular dependences of MEP in TMO. MEP was measured along the [001] axis. The field was applied in the (001) plane. $\varphi$ is the angle between the [010] axis and the field. Curves 1,2 , and 3 were measured at $T=4.2 \mathrm{~K}$ in various fields: curve 1 at $H=20 \mathrm{kOe} ; 2-40 \mathrm{kOe} ; 3-60 \mathrm{kOe}$. Curve 4 was measured at $T=0.39 \mathrm{~K}$ and $H=100 \mathrm{kOe}$.

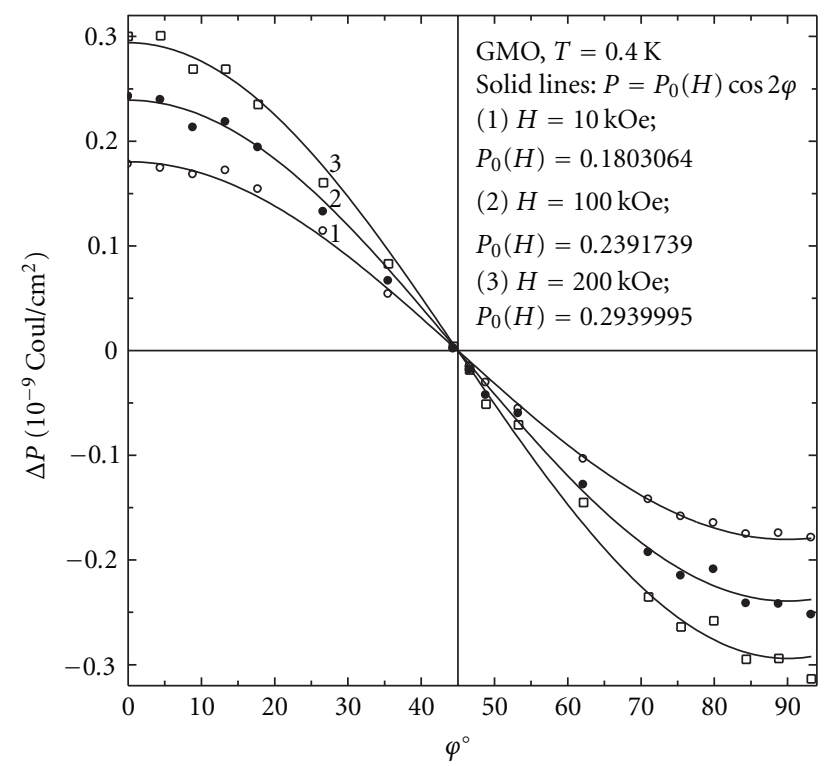

FIGURE 12: Angular dependences of MEP in GMO at various values of the magnetic fields. $\varphi^{\circ}$ is the angle between the [010] axis and the magnetic field. $T=0.4 \mathrm{~K}$. Curve (1) $H=10 \mathrm{kOe}$; (2) $100 \mathrm{kOe}$; (3) $200 \mathrm{kOe}$.

the ranges of variables $H=(0-200) \mathrm{kOe}$ and $T>0.4 \mathrm{~K}$ satisfactorily.

The experimental field dependences $\Delta P\left(H^{2}\right)$ in GMO at $T=4.2 \mathrm{~K}$ and $0<H<20 \mathrm{kOe}$ are shown in Figure 13 for two orientations of the field along the [010] axis (filled circles) and along the [100] axis (open circles). 


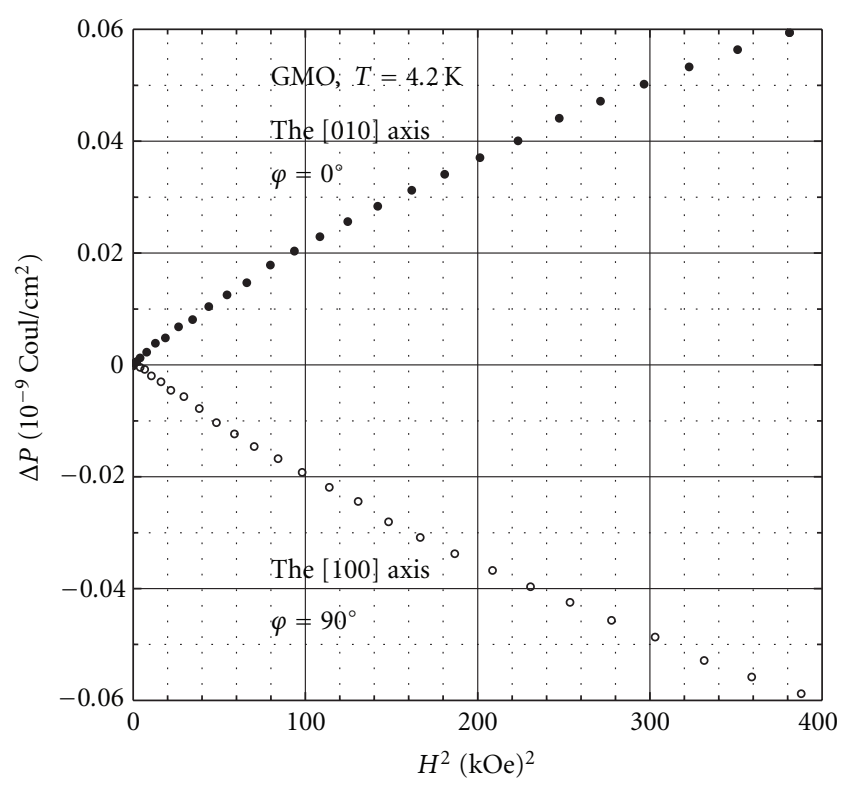

Figure 13: The dependences of MEP in GMO upon the magnetic field squared at $T=4.2 \mathrm{~K}$. Filled circles were obtained in the magnetic field along the [010] axis; open ones - the field along the [100] axis.

It is seen that, for both directions of the field, the experimental isotherms agree with the relation (28) only at $H^{2}<40(\mathrm{kOe})^{2}$, that is, $H<6.3 \mathrm{kOe}$. At higher fields, the disagreement increases. At $T=0.4 \mathrm{~K}$, the theory [36] fails utterly. So, the theory [36] is practically inapplicable to the description of MEE in GMO at low temperatures. The main origin of the magnetoelectric effect in rare earth molybdates is the single ion magnetostriction of rare earth ions $[37,38]$. So, we supposed that the experimental dependence $P(m)$ in GMO can be described by the relation analogous to that obtained in [49] for the single ion magnetostriction:

$$
\Delta P(m(H, T))=\Delta P_{0} \cdot \hat{I}_{l+1 / 2}\left[L^{-1}(m(H, T))\right] .
$$

Here, $\widehat{I}_{l+1 / 2}(x)$ is a normalized hyperbolic Bessel function of l-th order:

$$
\begin{gathered}
\hat{I}_{l+1 / 2}(x)=\left[I_{l+1 / 2}(x)\right] /\left[I_{1 / 2}(x)\right] \\
I_{l+1 / 2}(x)=\int_{-1}^{1} \mathrm{P}_{l}(y) \exp (x y) d y ;
\end{gathered}
$$

$\mathrm{P}_{l}(y)$ is the Legendre polynomial. $L^{-1}(m)$ is the inverse Langevin function of the reduced magnetization $m=M / M_{S}$. For the case considered, $l=2$ has been defined by the second-order symmetry of the angular dependence $P(\varphi)$ obtained from Figure 12. For $l=2$, the reduced hyperbolic Bessel function is given by the relation:

$$
\hat{I}_{5 / 2}(x)=1+\frac{3}{x^{2}}-\frac{3}{x} \operatorname{cth} x .
$$

Relation (32) was used to describe the field dependence of MEE in GMO. Results are shown on Figure 14. Points on this figure present experimental dependences of MEP in GMO upon the reduced magnetization $m$. The circles present MEP values measured in the field directed along the [010] axis. The squares correspond to the field along the [100] axis. Filled symbols refer to the temperature $T=0.4 \mathrm{~K}$, open symbols refer to $T=4.2 \mathrm{~K}$. The experimental values of MEP do not exceed $\Delta P \approx 0.3 \cdot 10^{-9} \mathrm{Coul} / \mathrm{cm}^{2}$.

Calculated dependences $\Delta P(m)$ are shown by solid lines in Figure 14 for $T=4.2 \mathrm{~K}$ (curves 1 and 2). Coefficients $\Delta P_{0}$ are $-0.18 \cdot 10^{-9} \mathrm{C} / \mathrm{cm}^{2}$ and $0.17 \cdot 10^{-9} \mathrm{C} / \mathrm{cm}^{2}$ for the $[100]$ and [010] axes, respectively. For $0<m<0.8$, the divergence between the experiment and the theory is $\delta(\Delta P) /(\Delta P)<$ 0.02 . So, the relation (32) obtained from the theory of the single ion magnetostriction agrees to the experiment well at $0<m<0.8$. For $m=0.95, \delta(\Delta P) /(\Delta P)=0.09$. The value $m=0.95$ in the case of GMO corresponds to the field $H \approx 56,7 \mathrm{kOe}$. It is seen that the range of validity of relation (32) is wider than relation (28) [36]. Nevertheless, the most part of the field range is not described.

The anomaly near $m=1$ indicates some additional effects that should be considered. The dependences $P(m)$ at $T=0.4 \mathrm{~K}$ cannot be explained by the theory of the single ion magnetostriction in its present form because this theory does not take account of the antiferromagnetic structure. The magnetic phase transition paramagnetismantiferromagnetism that takes place in GMO at $T=0.3 \mathrm{~K}$ may affect the results of the measurements at $T=0.4 \mathrm{~K}$. The solid lines 3 and 4 are drawn only for the eyes guide.

MEE in $\mathrm{Sm}_{2}\left(\mathrm{MoO}_{4}\right)_{3}(\mathrm{SMO})$ was investigated in direct current magnetic field up to $200 \mathrm{kOe}$ at temperatures from $4.4 \mathrm{~K}$ to $0.43 \mathrm{~K}[50]$. MEP was measured along the [001] axis in the magnetic field along the [010] axis and [100] axis.

Figure 15 represents a cycle of the MEP variation (abcde) observed in a single domain sample of SMO at $T=$ $1.4 \mathrm{~K}$ in the magnetic field directed along the 010] axis. At the ascending branch of the cycle (ab), the magnetic field was increased at a rate $d H / d t=0.755 \mathrm{kOe} / \mathrm{s}$. The branch ab was passed for $265 \mathrm{~s}$. It represents the experimental dependence of MEP in the increasing field $\Delta P_{e \uparrow}(H)$. At the b point $(H=200 \mathrm{kOe})$, the $\mathrm{MEP}$ was $\Delta P_{e \uparrow}=-5.46$. $10^{-9} \mathrm{Coul} / \mathrm{cm}^{2}$. At this point, the magnetic field was fixed and remained unvaried for $232 \mathrm{~s}$. During this time, the $\mathrm{MEP}$ relaxed to $\Delta P_{e \downarrow}=-3.01 \cdot 10^{-9} \mathrm{Coul} / \mathrm{cm}^{2}$ (point $\mathrm{c}$ ). At point $c$, the magnetic field started to decrease at a rate $d H / d t=-0.755 \mathrm{kOe} / \mathrm{s}$. The descending branch $\mathrm{cd}$ represents the experimental dependence of MEP in the decreasing field $\Delta P_{e \downarrow}(H)$. At point d, the magnetic field reached zero and was fixed. The MEP was found to be $\Delta P_{e \downarrow}=3.12 \cdot 10^{-9} \mathrm{Coul} / \mathrm{cm}^{2}$ at this point. After fixing the magnetic field at zero, the MEP was measured for $338 \mathrm{~s}$. During this time, it relaxed to $\Delta P=1.4 \cdot 10^{-9} \mathrm{Coul} / \mathrm{cm}^{2}$.

This variation of the MEP is plotted as de on Figure 15. Thus, the MEP in SMO relaxes in a fixed magnetic field. The relaxation is not due to the charge leakage through the electrometer input circuit because the times of measurements at a fixed field $232 \mathrm{~s}$ and $338 \mathrm{~s}$ are two orders of magnitude shorter than the time constant of the electrometer $\tau_{\mathrm{em}}>10^{4} \mathrm{~s}$. Analogous measurements of the MEP cycles were performed for other magnetic field 


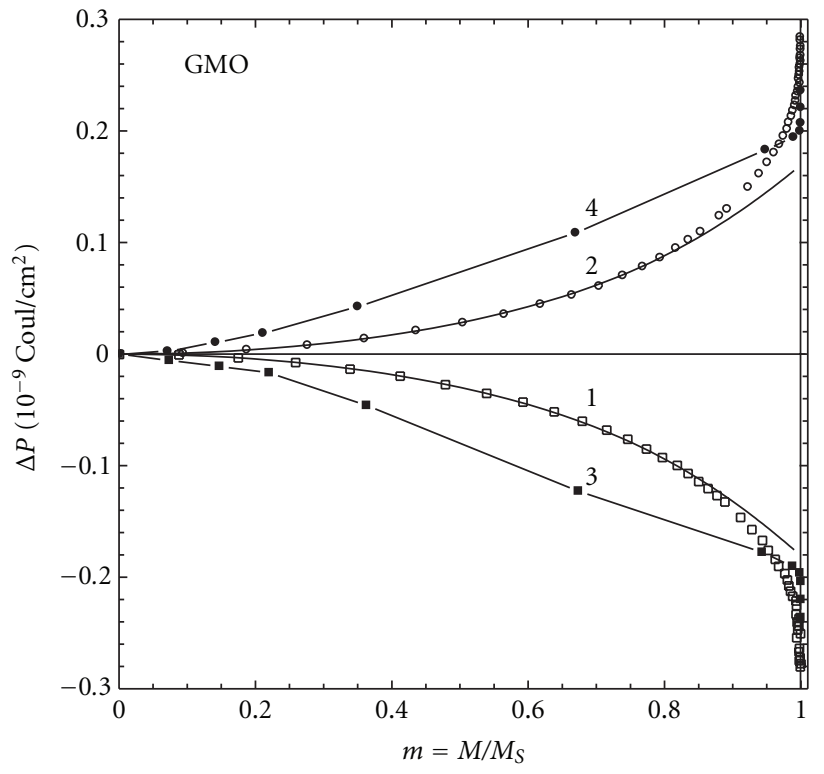

FIGURE 14: Dependences of MEP along the [001] axis in GMO upon the reduced magnetization $m=M / M_{\mathrm{S}}$ at various temperatures and magnetic field directions. $T=4.2 \mathrm{~K}$ : curve (1) the magnetic field along the [100] axis; curve (2) the field along the [010] axis. $T=$ $0.4 \mathrm{~K}$ : curve (3) the field along the [100] axis; curve (4) the field along the [010] axis.

orientations and temperatures. The time dependences of MEP on the segments bc and de were fitted well by the exponents:

$$
\Delta P_{e}(H=\text { const, } t)=\Delta P_{0} \exp \left(-\frac{t}{\tau}\right)+P_{R}
$$

The relaxation time $\tau$ increased with decreasing temperature. It depended upon the magnetic field only weakly.

Table 2 presents the values of $\tau$ obtained at various temperatures in the field $200 \mathrm{kOe}$.

The relaxation of the MEP noticeably affected the MEP field dependences because the measurement time $265 \mathrm{~s}$ was comparable with the relaxation time $\tau$. Our measurements showed that the relation between $\Delta P_{0}$ and $P_{R}$ can be approximately described by the expression $P_{R} \approx \beta\left(\Delta P_{0}\right)$ where $\beta \ll 1$ is a constant. In this case, a time-dependent contribution could be eliminated from the experimental field dependences $\Delta P_{e \uparrow \downarrow}(H)$ using the relations:

$$
\begin{aligned}
\Delta P_{\uparrow}(H)= & \Delta P_{e \uparrow}(H)+\int_{0}^{H} \frac{\Delta P_{e \uparrow}(H)}{\alpha \tau(1+\beta)} d H ; \\
\Delta P_{\uparrow}(H)= & \Delta P_{e \uparrow}(H)-\int_{H_{m}}^{H} \frac{\Delta P_{e \downarrow}(H)}{\alpha \tau(1+\beta)} d H \\
& +P_{\uparrow}\left(H_{m}\right)-P_{e \downarrow}\left(H_{m}\right) .
\end{aligned}
$$

Here, $P_{\uparrow \downarrow}(H)$ does not depend on the time. The $\uparrow$ and $\downarrow$ arrows denote the values obtained under increasing and decreasing field respectively; $H_{m}$ is the maximum field reached in the measurements; $\alpha=d H / d t$ is the rate of the
TABLE 2: Relaxation time $\tau$ of MEP in SMO at various temperatures in the field $200 \mathrm{kOe}$.

\begin{tabular}{lcc}
\hline$T(\mathrm{~K})$ & $\begin{array}{c}\text { The field along } \\
{[010], \tau(\mathrm{s})}\end{array}$ & $\begin{array}{c}\text { The field along } \\
{[100], \tau(\mathrm{s})}\end{array}$ \\
\hline 4.4 & 127 & 128 \\
2.6 & 279 & 192 \\
1.4 & 337 & 403 \\
0.9 & 490 & 490 \\
0.43 & 534 & 828 \\
\hline
\end{tabular}

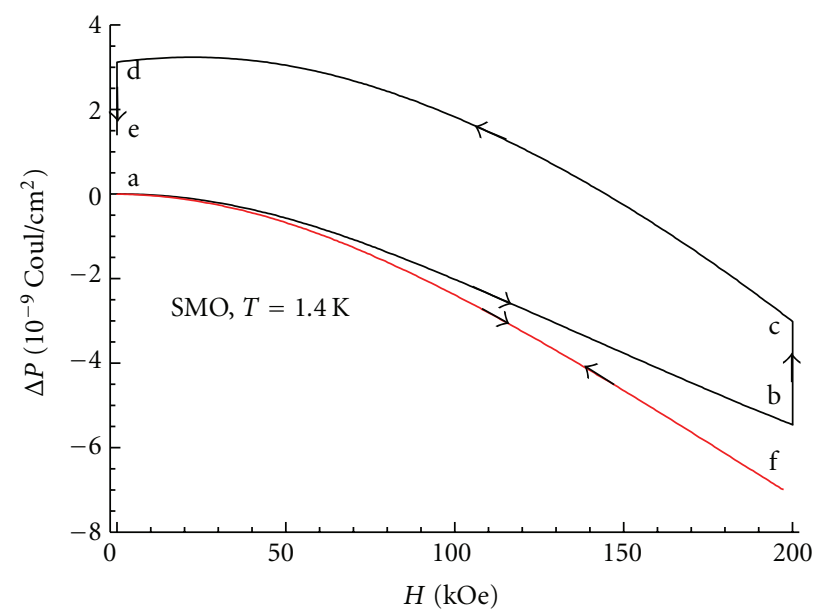

FIGURE 15: Field dependences of MEP in $\mathrm{Sm}_{2}\left(\mathrm{MoO}_{4}\right)_{3}$ at $T=1.4 \mathrm{~K}$. The field is directed along the [010] axis. Curves ab and cd present experimental dependences $\Delta P_{e \uparrow \downarrow}(H)$ in increasing and decreasing field, respectively. Straight lines bc and de present the relaxation of $\Delta P$ with the time at $H=200 \mathrm{kOe}$ and $H=0$, respectively. Curves af and fa are calculated from the curves ab and cd using (36) and (37), respectively.

magnetic field variation with the time; $P_{\uparrow}\left(H_{m}\right)$ is the true MEP at the maximum field calculated from the experimental curve $P_{e^{\dagger}}(H)$ using (36); $P_{e^{\dagger}}\left(H_{m}\right)$ is the experimental value of MEP at the start of the decrease in the field (point $c$ in the cycle in Figure 15). Curves $a b$ and cd were used to obtain the af and fa curves (Figure 15) by means of (36) and (37), respectively. One can see that elimination of the time-dependent contribution from the experimental $\Delta P_{e \nmid \downarrow}(H)$ dependences yields a hysteresis-free $P(H)$ relation. Analogous results were obtained at the temperatures $0.9 ; 1.4$; $2.6 ; 4.4 \mathrm{~K}$ in the field along the [010] and [100] axes.

Corresponding curves are shown in Figure 16. The dependences 1 and 6 obtained at $4.4 \mathrm{~K}$ are fitted well by the relation (28) at $\varphi=0 ; \pi / 2$ and $H<50 \mathrm{kOe}$. At higher fields and lower temperatures, this relation fails.

The data described in Section 4 qualitatively confirm the magnetostriction mechanism of MEE in rare earth molybdates. This mechanism is based on the assumption that the magnetostriction affects the electric polarization of these ferroelectric compounds.

Table 3 shows the values of MEP in the three rare earth molybdates investigated here at fixed temperature and field values. The values of $g$ and $J$ were taken from [51]. There is a clear qualitative correlation between the values of the angular 
TABLe 3: A comparison of MEP absolute values in TMO, GMO, and SMO at $T=4.2 \mathrm{~K}$ and $H=100 \mathrm{kOe}$.

\begin{tabular}{|c|c|c|c|c|c|}
\hline Compound & Figure & $T(\mathrm{~K})$ & $\Delta P\left(10^{-9} \mathrm{Coul} / \mathrm{cm}^{2}\right)$ & $L$ of $\mathrm{R}^{3+}$ ion & $g J$ of $\mathrm{R}^{3+}$ ion \\
\hline TMO & Figure 11 & 0.39 & $\approx(42-57)$ & 3 & 9 \\
\hline GMO & Figure 12 & 0.4 & $\approx 0.25$ & 0 & 7 \\
\hline SMO & Figure 16 & 0.43 & $\approx 3$ & 5 & 0.7 \\
\hline
\end{tabular}

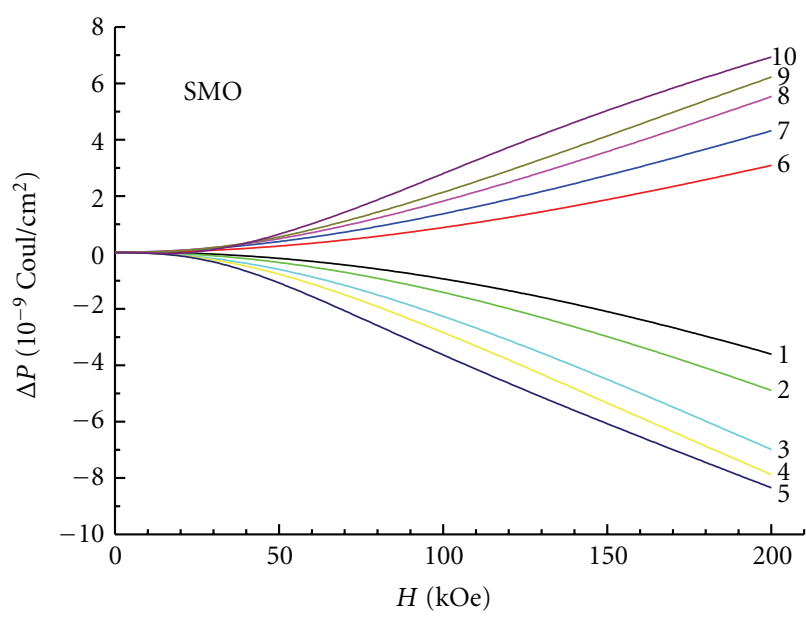

FIGURE 16: Field dependences of MEP in SMO after the elimination of the time-dependent contribution. Curves 1-5 are measured in the field along the [010] axis; curves $6-10$ are measured in the field along the $[100]$ axis. The temperatures: curves $(1,6) T=4.4 \mathrm{~K} ;(2$, 7) $2.6 \mathrm{~K} ;(3,8) 1.4 \mathrm{~K} ;(4,9) 0.9 \mathrm{~K} ;(5,10) 0.43 \mathrm{~K}$.

momentum $L$ and the values of $\Delta P$. GMO with zero angular momentum of $\mathrm{R}^{3+}$ ion possesses the least $\Delta P$ value. The angular momentum $L$ characterizes the spatial anisotropy of an ion. It is obvious that the rotation of an anisotropic ion with $L \neq 0$ produces more strains in a crystal lattice than the rotation of a spherically symmetrical ion with $L=0$. So, the change of the electric polarization $(\mathrm{MEP}=\Delta P)$ is larger for $L \neq 0$ than for $L=0$. There is some incongruity in this conclusion: the angular momentum of $\mathrm{Sm}^{3+}$ ion is larger than that of $\mathrm{Tb}^{3+}$ ion, but the $\Delta P$ value in SMO is the order of magnitude smaller than that in TMO. This fact can be explained qualitatively by relations between the ionic magnetic moments $g J \mu_{B}$ of $\mathrm{R}^{3+}$ ions under consideration. Here, $g$ is $g$-factor; $J=L \pm S$ is the total mechanical moment of $\mathrm{R}^{3+}$ ion; $S$ is the spin mechanical moment of $\mathrm{R}^{3+}$ ion. The sign "-" corresponds to light rare earth ions from Ce to Eu, and the sign "+" corresponds to heavy rare earth ions from $\mathrm{Tb}$ to $\mathrm{Yb} . \mu_{B}$ is the Bohr magneton. One can see that the magnetic moment of $\mathrm{Tb}^{3+}$ ion is larger than that one of $\mathrm{Sm}^{3+}$ ion. So, one should conclude that the total effect of the space anisotropy and the magnetic moment of $\mathrm{Tb}^{3+}$ ion is larger than that one of $\mathrm{Sm}^{3+}$ ion. It can be because of that the ion with the larger magnetic moments rotates by the larger angle than the ion with a smaller magnetic moment in the same magnetic field. The final result is determined by the quantitative contributions of the spatial anisotropy of ions and the angles of their rotations due to the interaction of their magnetic moments with the applied magnetic field.

\section{Magneto-Optical Properties}

In [26], the experimental dependences of the magnetization in terbium molybdate $\beta^{\prime}-\mathrm{Tb}_{2}\left(\mathrm{MoO}_{4}\right)_{3}$ (TMO) upon the magnetic field and the temperature $M(H, T)$ were described satisfactorily using the theory of the magnetism of singlets [31] at low temperatures $T<30 \mathrm{~K}$. At higher temperatures, the agreement was not good. It was due to increasing population of highest levels of ${ }^{7} \mathrm{~F}_{6}$ multiplet of $\mathrm{Tb}^{3+}$ ions. For the calculation of the magnetization at high temperatures, the highest levels of ${ }^{7} \mathrm{~F}_{6}$ multiplet should be known. To calculate the spectrum of ${ }^{7} \mathrm{~F}_{6}$ multiplet, one should know the crystal field acting on $\mathrm{Tb}^{3+}$-ion. Now, there is no theory that enables one to calculate the crystal field parameters from the first principles. So, the investigations that allow obtain directly the data about the spectra of multiplets are very useful. For example, the knowledge of the spectrum of the excited multiplet ${ }^{5} \mathrm{D}_{4}$ can simplify the calculations of the crystal field parameters in the case under investigation. The spectrum of ${ }^{5} \mathrm{D}_{4}$ multiplet can be found from the experimental data for the optical absorption in TMO at low temperatures. The optical absorption in TMO was studied at zero magnetic field in [52]. 14 well-resolved absorption peaks were observed. It was established that those peaks correspond to the transitions ${ }^{7} \mathrm{~F}_{6} \Rightarrow{ }^{5} \mathrm{D}_{4}$ in $\mathrm{Tb}^{3+}$ ions.

In [53], the absorption spectra were measured in TMO in the nonpolarized light in the magnetic field $H \leq 200 \mathrm{kOe}$ along the [001] and [110] axes at $T=4.2 \mathrm{~K}$ and $T=1.7 \mathrm{~K}$. A wave vector of the light was parallel to the magnetic field. At zero field, 14 well-resolved peaks with wave numbers $\nu=20519 \mathrm{~cm}^{-1}-20655 \mathrm{~cm}^{-1}(\lambda=4873.5 \AA-4841.4 \AA)$ were observed. The magnetic field shifted the spectrum toward large $v$ values. The spectra were measured at 29 different field values. The experimental field dependences of the wave numbers of the absorption peaks $v_{i}(H)$ were obtained for both directions of the field.

The spectra did not depend upon the temperature. There was a pronounced dependence of the peak numbers and of the rate of their shift with the field $d v_{i} / d H$ upon the direction of the field. The analysis of these dependences showed that the excited multiplet ${ }^{5} \mathrm{D}_{4}$ of $\mathrm{Tb}^{3+}$ ion in the crystal field of TMO could be considered as consisting of five singlets and two quasidoublets. On the base of the measurements and analysis of the absorption spectra in terbium molybdate, the field dependence of the energy spectrum $E_{i}(H)$ of the excited multiplet ${ }^{5} \mathrm{D}_{4}$ of $\mathrm{Tb}^{3+}$ ion in the crystal field with the point group symmetry $\mathrm{C}_{2}$ was found for the magnetic field up to $200 \mathrm{kOe}$ along the [001] axis. The experimental dependences $E_{i}(H)$ could be described well by the theory of the magnetism of singlets. 
The number of the absorption peaks was explained by the existing of two types of nonequivalent sites for $\mathrm{Tb}^{3+}$ ions in the crystal lattice of TMO.

\section{Anomalously High Photo Voltages}

In [54], an anomalously high photoinduced voltage (APV effect) in TMO was observed. The illumination of a single crystal of TMO by the beam of the argon laser with a wave length of $\lambda=4880 \AA$ and a power of $0.5 \mathrm{~W}$ induced in the ferroelectric single domain sample an additional electric polarization. The wave length corresponded to a proper absorption band of TMO. The laser beam was directed along the [110] axis. The surface of polarization of the laser beam was normal to the (001) plane. The direction of the photoinduced polarization was the same as that of the spontaneous polarization. The laser beam was focused to a spot with an area $10^{-2} \mathrm{~cm}^{2}$. It means that the distribution of the intensity of the light was highly inhomogeneous. The average value of the intensity in the illuminated spot was $\approx 50 \mathrm{~W} / \mathrm{cm}^{2}$. The photoinduced electric polarization of the crystal depended upon the time of the illumination. After the illumination during $4.5 \mathrm{~h}$, the photoinduced polarization was approximately three times larger than the spontaneous electric polarization and showed the tendency to the saturation.

The laser beam with the wave length $4880 \AA$ induced in TMO the luminescence in the green part of the visible spectrum with the wave length $\approx 5430 \AA$. The quantum output constituted $20 \%$.

In [55], the dependences of the photoinduced voltage in TMO upon the time $t$ of the illumination by a laser beam were measured at room temperature at $0.1 \mathrm{~s} \leq$ $t \leq 4500 \mathrm{~s}$. The power of the laser radiation was $0.4 \mathrm{~W}$. The distribution of the intensity along the sample was varied from highly inhomogeneous to homogeneous. It was found that the photoinduced voltages of two types with opposite signs appear due to the inhomogeneous illumination.

In [55], the APV effect in TMO was measured at heightened sensibility, respectively, to the photoinduced electric polarization of the crystal.

Due to this, it was possible to observe relatively small contribution to the photoinduced polarization $\Delta P_{\mathrm{ph}}$ that was directed opposite to the spontaneous electric polarization $P_{S}$. This effect was observed at a small duration of illumination $t<80 \mathrm{~s}$. It was called APV-1. The effect that was observed in [54] was called APV-2. The sign of APV-1 was opposite to the sign of APV-2. The voltage and the relaxation time of APV1 were essentially smaller than those of APV-2. That is why the APV-1 effect was not observed on the background of the APV-2 effect in [54].

The first observation of APV-effect was reported for a single crystal sample of ferroelectric $\mathrm{SbSI}_{0.35} \mathrm{Br}_{0.65}$ [56]. A phenomenological theory of the APV effect was described in [57]. In works published up to now, the diffusion contribution to the photoinduced current was ignored $[57,58]$. According to [57], the time dependences of the photoinduced current and the photoinduced voltage are given by the following expressions:

$$
\begin{gathered}
i(t)=i_{0} \exp \left(-\frac{t}{\tau}\right) \\
V(t)=V_{0}\left[1-\exp \left(-\frac{t}{\tau}\right)\right] .
\end{gathered}
$$

The effect APV-1 is well described by the expressions (38) and (39). The effect APV-2 does not follow these expressions.

Figure 17 shows experimental time dependences of the photoinduced current $i(t)$ (curve 1) and the photoinduced voltage $V(t)$ (curve 2).

These curves were obtained on the sample number 1 with size $6 \times 7 \times 1 \mathrm{~mm}^{3}$. The large face of the sample was parallel to the plane (001).

The [100] and [010] crystal axes were directed along the bisectors of the right angles of the (001) sides. The laser beam was focused on the $7 \times 1 \mathrm{~mm}^{2}$ face of the sample by a cylinder lens to the spot with dimensions $2 \times 0.5 \mathrm{~mm}^{2}$. The large size of the spot was along the [001] axis of the crystal. The intensity of the light in the spot was $\approx 40 \mathrm{~W} / \mathrm{cm}^{2}$.

Arrows show the time when the light was switched on and off. These dependences are in a good agreement with expressions (38) and (39) respectively. The constants are $i_{0} \approx 0.2 \cdot 10^{-9} \mathrm{~A} ; V_{0} \approx-10 \mathrm{~V} ; \tau \approx 5 \mathrm{~s}$. The stepped form of the curve 1 is due to the resolution of the equipment. When the light is switched on, the negative voltage $V(t)$ appears. This voltage is due to the photoinduced electric polarization $\Delta \vec{P}_{\mathrm{ph}}$ that is directed opposite to the spontaneous electric polarization $\vec{P}_{S}$. The value $V_{0} \approx-10 \mathrm{~V}$ corresponds to the decreasing of $\vec{P}_{S}$ by the value $\Delta P_{\mathrm{ph}}=4.8 \cdot 10^{-9} \mathrm{C} / \mathrm{cm}^{2}$. When the light is switched off, the photoinduced voltage decreases exponentially with the time constant $\tau \approx 5 \mathrm{~s}$. The photoinduced voltage and the photoinduced current on Figure 17 are due to the effect APV-1.

Figure 18 shows the dependences $\Delta P_{\mathrm{ph}}(t)$ for various distributions of the light intensity along the sample. The switching on and off the light are shown by the arrows. Curve 1 was measured on the sample number 1 at the same conditions as in Figure 17. One can see that the type of the dependence shown in Figure 17 occurs only during short time of $\approx 200 \mathrm{~s}$ after the light is switched on. At $t \approx$ $(250--300) \mathrm{s}$, the $\Delta P_{\mathrm{ph}}(t)$ value reaches a minimum of $\Delta P_{\mathrm{ph}} \approx-4.8 \cdot 10^{-9} \mathrm{C} / \mathrm{cm}^{2}$ and begins to increase with increasing $t$.

At $t \approx 1700 \mathrm{~s}$, the sign of $\Delta P_{\mathrm{ph}}(t)$ is changed. The positive sign of $\Delta P_{\mathrm{ph}}(t)$ at $t>1700 \mathrm{~s}$ corresponds to the coincidence of the directions of $\vec{P}_{S}$ and $\overrightarrow{\Delta P}$ ph. The tendency to the saturation of $\Delta P_{\mathrm{ph}}(t)$ is seen at large values of $t$. At switching off the light, the positive jump of the photoinduced polarization occurs. It is of the same absolute value as the negative jump at switching on the light. After this jump, the photoinduced polarization continues to increase slowly: an after-effect takes place. In other words, the long negative pulse of the polarization due to the effect APV-1 exists on the background of slow positive drift of the polarization. This drift is due to the effect APV-2. 


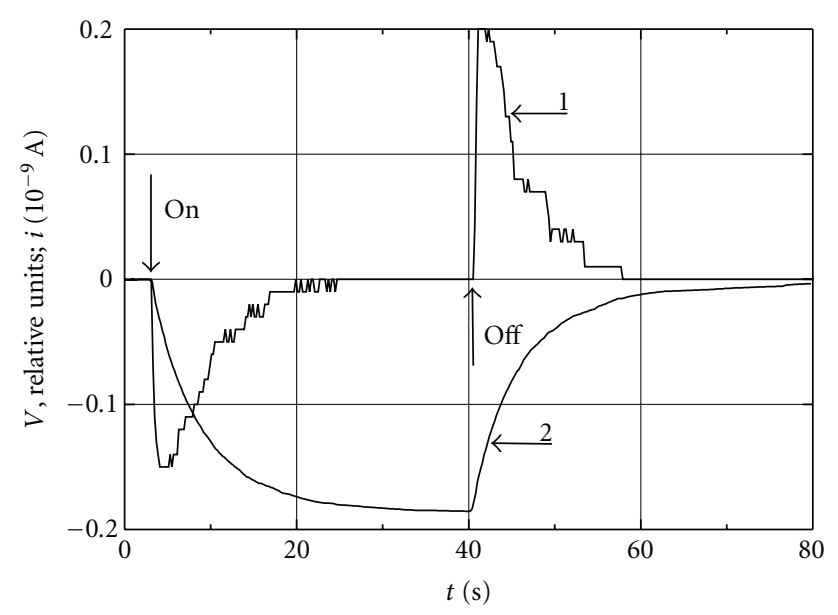

Figure 17: The dependence upon the time of the photoinduced current (curve 1) and the photoinduced voltage (curve 2) in TMO. Sample number 1 . The cross-section of the beam has size $0.5 \mathrm{~mm} \times$ $2 \mathrm{~mm}$.

Curve 2 in Figure 18 was measured on the sample number 1 with a homogeneous distribution of the intensity across the $7 \times 1 \mathrm{~mm}^{2}$ face of the sample. The cross-section of the laser beam had a circular form with diameter $\phi=7 \mathrm{~mm}$. So, the intensity of the illumination was lower than for curve 1 , and the photoinduced polarization was smaller. One can see that curve 2 shows a smaller relative contribution of the slow positive drift to the resulting polarization than curve 1 . It should be pointed out that in spite of the homogeneous illumination of the $7 \times 1 \mathrm{~mm}^{2}$ side the distribution of the intensity of the light was not homogeneous inside the sample along the direction of the propagation of the light due to the absorption. To obtain the $\Delta P_{\mathrm{ph}}(t)$ dependence at still more homogeneous illumination, the measurements were performed on the sample number 2. The sample number 2 was a cube of the size of $2 \times 2 \times 2 \mathrm{~mm}^{3}$. The relative directions of the crystal axes and of the laser beam were the same as for sample number 1 . The cross-section of the beam was as for curve 2 .

As the illuminated face of the sample number 2 was smaller than that for sample number 1 , the $\Delta P_{\mathrm{ph}}(t)$ value also was smaller. As the size of the sample number 2 along the direction of the propagation of the light was three times smaller than for the sample number 1 , the relative nonhomogeneity of the illumination was also smaller. Curve 3 in Figure 18 was measured on the sample number 2. One can see that in this case the change of the sign of the voltage was not observed during all the time of the illumination. At these conditions, the positive drift due to APV-2 is so small that one can neglect it.

Figure 19 shows curve 1 from Figure 18 contracted along the $t$-axis and its prolongation for large $t$ values that describes the relaxation of the photopolarization. The relaxation curve gives qualitative information that is important for explanation of the APV effect in TMO. The curve shows a very durable aftereffect. The increase of the photoinduced voltage continues during $\approx 3500 \mathrm{~s}$ after the light is switched

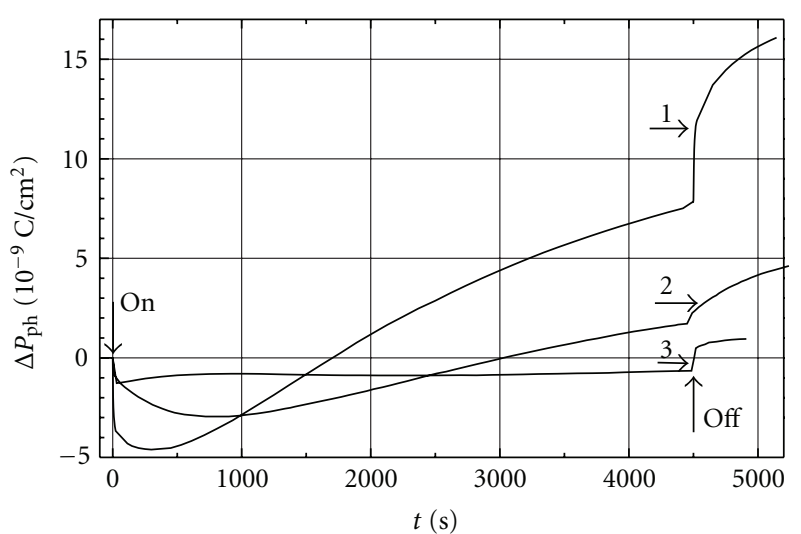

FIgure 18: The dependence of the photoinduced electric polarization in TMO upon the time. Curve 1. Sample number 1. A nonhomogeneous illumination. Curve 2. Sample number 1. A homogeneous illumination of the face of the sample. A nonhomogeneous distribution of the intensity in the sample due to the absorption. Curve 3. Sample number 2. An approximately homogeneous distribution of the intensity in the sample.

off. The voltage reaches the maximum $V_{\max } \approx 35 \mathrm{~V}$ at $t \approx$ $8000 \mathrm{~s}$ and then decreases.

The value of $V_{\max } \approx 35 \mathrm{~V}$ corresponds to the photopolarization $\Delta P_{\mathrm{ph} 2} \approx 17 \cdot 10^{-9} \mathrm{C} \cdot \mathrm{cm}^{-2}$. The relaxation time is $\tau_{\text {relax }} \approx 20 \mathrm{~h}=7.2 \times 10^{4} \mathrm{~s}$. It is more than the time constant of the input circuit of the electrometer $\tau_{\text {input }}$. Due to this, the dependence $V(t)$ of the relaxation process is not reproduced correctly. So, only the initial part of the relaxation curve is shown in Figure 19. The complete relaxation of the APV2 effect for the present experiment was achieved at $t_{R} \approx$ $150 \mathrm{~h}=5.4 \times 10^{5} \mathrm{~s}$. Due to the relation between $\tau_{\text {input }}$ and $\tau_{\text {relax }}$, this value of $t_{R}$ should be considered as a bottom estimate.

The two effects described above show quite different dependences upon the conditions of the experiment.

The APV-1 effect exists both at homogeneous and nonhomogeneous illuminations (Figure 18). It reaches the saturation at $t \approx 40 \mathrm{~s}$ (Figure 17). The APV-2 effect exists only at nonhomogeneous illumination.

It was not observed at a homogeneous illumination (Figure 18, curve 3 ). It depends on the time and the geometry of the beam cross-section (Figure 18).

The time constant of the APV-1 effect is $\tau=5 \mathrm{~s}$. From Figures 17 and 18, one can see that APV-1 effect reaches the saturation in a relatively short time and after this the voltage due to APV-1 effect is constant. Contrary to APV-1 effect, the voltage due to APV-2 effect grows for a very long time of the order of several thousand seconds showing only very weak tendency to the saturation.

The APV-2 effect possesses the after-effect: the $P_{\text {ph2 }}$ value increases during several thousand seconds after the light is switched off (Figure 19). No after-effect was observed while measuring the APV-1 effect.

The APV-1 effect has a feature that is common to photo-induced currents measured in [59-62]. It is a strong correlation between the switching the light off and the change of the direction of the photoinduced current. The 
photoinduced voltage due to this effect begins to decrease immediately after the light is switched off. At the same time, the direction of the corresponding current is changed. The APV-2 effect is essentially different from those observed in [59-62]. The photoinduced voltage due to this effect is growing during a long time (almost an hour) after the light is switched off. The photoinduced current due to this effect changes its sign with a very long delay after the light is switched off. So, it is ascertained that there are two different effects: APV-1 and APV-2. They have quite different mechanisms.

In [55], it was concluded that the APV-1 effect originates due to the transitions of $\mathrm{Tb}^{3+}$ ions from the ground state ${ }^{7} \mathrm{~F}_{6}$ to the excited state ${ }^{5} \mathrm{D}_{4}$ induced by the laser irradiation. The saturation of the APV-1 effect is reached when the dynamic equilibrium between the numbers of straightforward and backward transitions is established. The lattice accommodation explains the large value of the time constant $\tau \approx 5 \mathrm{~s}$ of the APV-1 effect. The process of the accommodation takes some time. That is why the time constant $\tau$ is essentially larger than the life time of a free $\mathrm{Tb}^{3+}$ ion in the excited state ${ }^{5} \mathrm{D}_{4}$.

The APV-2 effect originates from the autoionization of the excited $\mathrm{Tb}^{3+}$ ions and the subsequent diffusion of the knocked out electrons in the electric field of the spontaneous polarization through the crystal. The autoionization can be caused by the internal electrical field related to the ferroelectric order. The autoionization results in appearance of a $\mathrm{Tb}^{4+}$ hole and a nonequilibrium electron. If the light is nonhomogeneous, the diffusion of these carriers from illuminated part of the sample to the nonilluminated one gives rise to the photoinduced current. The sign of the effect is determined by the fact that a pair $\mathrm{Tb}^{4+}-\left(\mathrm{MoO}_{4}\right)^{2-}$ appears instead of a pair $\mathrm{Tb}^{3+}-\left(\mathrm{MoO}_{4}\right)^{2-}$. So, autoionization increases the resulting electrical dipole moment, and the polarization of the sample increases as well.

\section{Fluctuations Near the $\beta-\beta^{\prime}$ Phase Transition}

In [63], giant fluctuations of the amplitude of the acoustic resonant peak were observed experimentally in orthorhombic $\mathrm{Gd}_{2}\left(\mathrm{MoO}_{4}\right)_{3}(\mathrm{GMO})$ near the Curie temperature. The temperature dependence of the intensity of the acoustic main resonant peak was measured in the temperature range $(22-165)^{\circ} \mathrm{C}$ including the Curie temperature, $T_{C}=159^{\circ} \mathrm{C}$. The low-frequency fluctuations of the amplitude of the resonant current through the sample were observed in the vicinity of the Curie temperature. The sample was cut in the (001) plane. Its dimensions were $7 \times 7 \times 1 \mathrm{~mm}^{3}$. The diagonals of the (001) face were parallel to the orthorhombic $[100]$ and $[010]$ axis.

The piezo-acoustic resonance was investigated. The scheme recommended in the list of standards on measurements of piezo-electric crystals [64] was used. Acoustic oscillations in the sample were generated using self-exciting method. An oscilloscope was used as a detector of oscillations in the sample. Frequency dependences of the amplitude of the sinusoidal input voltage of the oscilloscope $V_{1}$ were

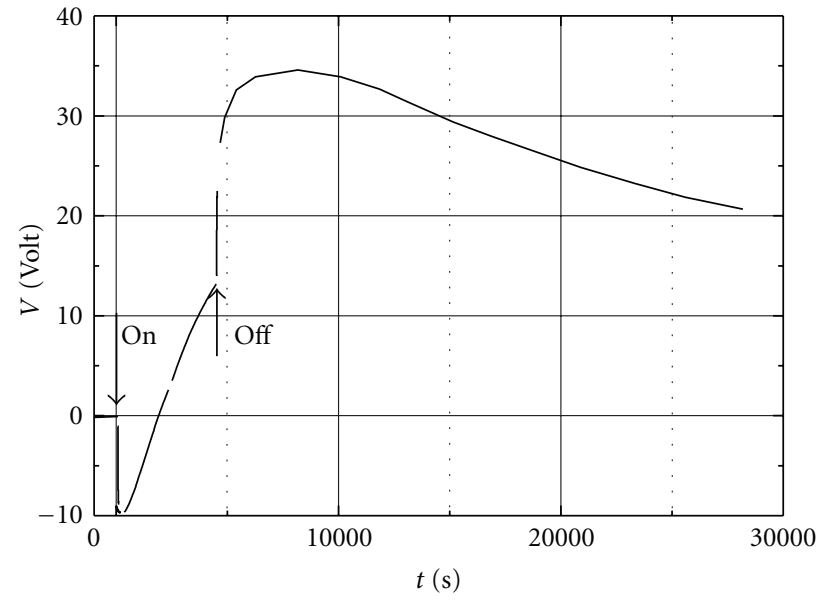

FIGURE 19: The relaxation of the photoinduced voltage in terbium molybdate after measuring curve 1 on Figure 18.

measured at fixed amplitude of the output voltage of the generator $V_{0}$ in the frequency range $(20-300) \mathrm{kHz}$ at $T=$ $(22-165)^{\circ} \mathrm{C}$. Under the influence of an alternative voltage, the alternative strains arise in a piezoelectric crystal as a consequence of the inverse piezoe-ffect. Under the influence of these strains, the alternative electric polarization arises in the crystal as a consequence of the direct piezoe-ffect. This alternative polarization is equivalent to appearing of an additional alternative electric current. The dependence of this additional current upon the frequency and the value of the voltage of the generator is described in terms of an equivalent electrical oscillating contour. This contour consists of an active resistance $R$, an inductivity $L$, and a capacity $C$ that are connected in series $[65,66]$. The capacity $C$ depends upon the elastic and piezoelectric properties of the crystal, its dielectric permittivity, the dimensions of the sample, and the oscillation mode. The inductivity $L$ depends upon the crystal density, its piezoelectric constants and the dimensions of the sample. The resistance $R$ is determined by the dissipation of the oscillation energy due to the internal friction and depends on the viscosity, the piezoelectric constants, and the dimensions of the sample.

The equivalent contour, the static resistance of the sample $R_{1}$ (the resistance in the absence of the oscillations), and the static capacity of the sample $C_{1}$ (the capacity in the absence of the oscillations) are connected in parallel. When the generator frequency is equal to the resonant frequency of the equivalent contour, the impedance of the contour decreases and becomes equal to its active resistance $R$. The decreasing of the sample impedance results in an increase of the current through the sample. This results in an increase of the input voltage of the oscilloscope $V_{1}$, and the resonant maximum appears on the frequency dependence $V_{1}(f)$ at

$$
f=f_{R}=\frac{1}{2 \pi \sqrt{L C}} .
$$


The value of the maximum is given by the following equation:

$$
V_{1}\left[f_{R}(T)\right]=V_{R}(T)=\frac{V_{0}\left[1+\xi(T) C_{1}^{2}\right]}{\sqrt{\xi(T) C_{2}^{2}+\left[1+\xi(T) C_{1}\left(C_{1}+C_{2}\right)\right]^{2}}} .
$$

Here, $T$ is the temperature; $C_{1}$ the static capacity of the sample; $C_{2}$ the input capacity of the oscilloscope;

$$
\xi(T)=\frac{[R(T)]^{2}}{L(T) C(T)}=4 \pi^{2}\left[f_{R}(T)\right]^{2}[R(T)]^{2} ;
$$

$R, L, C$ are the resistance, the inductivity, and the capacity of the equivalent contour, respectively. Equation (41) is derived for the case $R_{1} \gg 1 /\left(\omega C_{1}\right) ; R_{2} \gg 1 /\left(\omega C_{2}\right) . R_{2}$ is the active input resistance of the oscilloscope. It was assumed that the values $R, C_{1}$, and $C_{2}$ do not shift noticeably the resonance term $\omega^{2} L C=1$.

Figure 20 shows the experimental temperature dependence of the resonant peak intensity $V_{R}(T)$.

At temperatures higher than $\approx 130^{\circ} \mathrm{C}$, the intensity $V_{R}(T)$ displays large and slow fluctuations at constant values of the temperature and the frequency. These fluctuations can be fixed visually.

The time of fluctuations reaches tens of seconds. Figure 20 shows maximum (open circles) and minimum (filled circles) values of $V_{R}$. The maximum and the minimum values of $V_{R}$ were found from the observation during two minutes at fixed values of the temperature and the frequency. The variations of $V_{R}$ due to the fluctuations reached 0.1 Volt. The fluctuations could be observed only in the limit of the resonant peak width $\Delta f_{R} \approx 1 \mathrm{kHz}$. Out of the resonance, they were zero with the accuracy of $0.008 V_{R} \approx 0.0003$ Volt. Curves are drawn only for the eyes guide.

Figure 21 shows the time dependence of the resonant peak intensity $V_{R}(t)$ at $T=154.25^{\circ} \mathrm{C}$ and $f_{R}=154.9 \mathrm{kHz}$. The oscillating line is a spline. The horizontal line is a mean value of $V_{R}$ over the time of observations. The mean square fluctuation of $V_{R}$ is $\approx 33 \mathrm{mV}$.

It constitutes $\approx 15 \%$ of the mean value $\bar{V}_{R} \approx 0.22$ Volt. The real frequency spectrum of the fluctuations is wider than that of the curve on Figure 21. Rapid fluctuations occur with periods much less than one second. So fast variations of $V_{R}(t)$ can be seen but it is impossible to fix them visually. Observed fluctuations of $V_{R}(t)$ occurs due to the fluctuations of the resonant current through the sample. When the temperature of the sample is near the temperature of the phase transition, various parameters of the crystal fluctuate and lead to the fluctuations of the impedance of the equivalent contour. This results in the fluctuations of the current through the sample. The value $V_{R}$ at the resonance is related to the parameters of the equivalent contour by (41). It is seen from (41) that the value of $V_{R}$ and consequently the values of its fluctuations can be presented as a function of the only variable $\xi=R^{2} / L C$. It can be shown that $\xi$ depends on four material constants. They are the elastic constant, the dielectric permeability, the coefficient of the internal friction, and the piezoelectric constant. $\xi$ depends

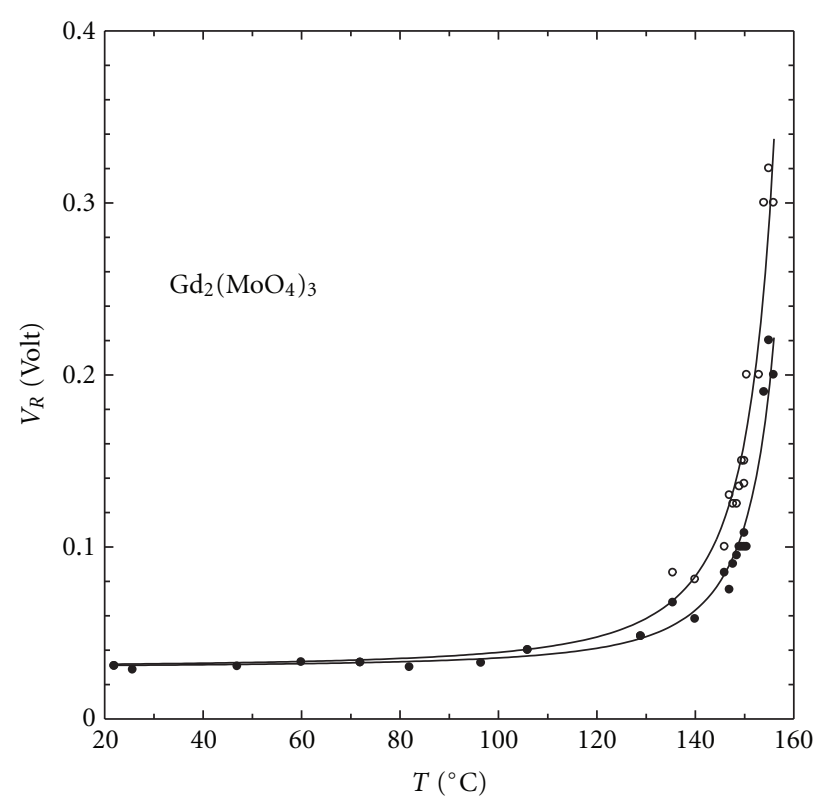

FIGURE 20: The temperature dependences of the maximum (open circles) and minimum (filled circles) intensities of the fluctuating resonant peak $V_{R}$. Lines are drawn for the eyes guide.

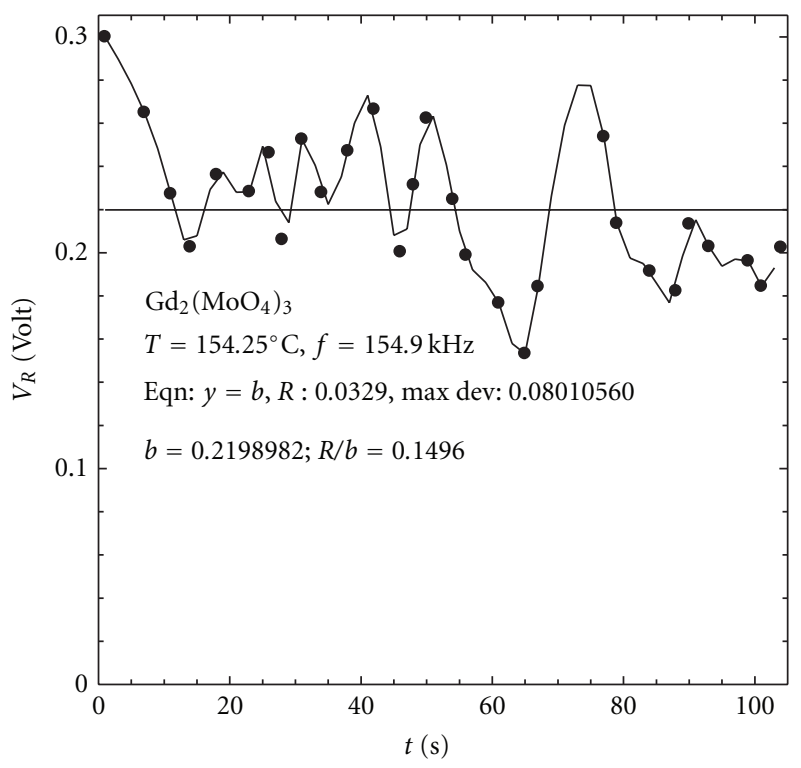

Figure 21: Fluctuations at $T=154.25^{\circ} \mathrm{C}$ and $f_{R}=154.9 \mathrm{kHz}$. The oscillating line is a spline. The horizontal line is a mean value of $V_{R}$ over the time of observations.

also upon the density $\rho$, but fluctuations of the density cannot reach so large values as shown on Figure 21. The observed fluctuations of the piezoacoustic impedance in GMO exceeds fluctuations of parameters of a crystal reported up to now [67] by the two orders of magnitude.

\section{Acknowledgments}

A. Zhukov wishes to underline that most scientific contribution in the field of RMO belongs to Prof. B. K. Ponomarev, 
who recently passed away. Consequently, the role of A. Zhukov, apart of magnetic measurements performed in 1990s, was mostly the preparation of this publication. This work was supported by EU ERA-NET Program under Project "SoMaMicSens" (MANUNET-2010-Basque-3), by Spanish Ministry of Science and Innovation, MICINN under Project MAT2010-18914 and by the Basque Government under Saiotek 11 MIMAGURA Project (S-PE11UN087). A. Zhukov wishes to acknowledge the support of the Basque Government under Program of Mobility of the Investigating Personnel of the Department of Education, Universities and Investigation, Grant nos. MV-2009-2-21 and MV-2010-231.

\section{References}

[1] L. H. Brixner, J. R. Barkley, and W. Jeitschko, "Rare-earth molibdates (VI)," in Handbook on the Physics and Chemistry of Rare Earths, K. A. Gschneidner Jr. and L. Eyring, Eds., vol. chapter 30, pp. 610-655, North-Holland Publishing Company, 1979.

[2] E. T. Keve, S. C. Abrahams, and J. L. Bernstein, "Ferroelectric ferroelastic paramagnetic beta- $\mathrm{Gd}_{2}\left(\mathrm{MoO}_{4}\right)_{3}$ crystal structure of the transition-metal molybdates and tungstates. VI," The Journal of Chemical Physics, vol. 54, no. 7, pp. 3195-3206, 1971.

[3] K. Ullakko, J. K. Huang, C. Kantner, R. C. O'Handley, and V. V. Kokorin, "Large magnetic-field-induced strains in Ni2MnGa single crystals," Applied Physics Letters, vol. 69, no. 13, pp. 1966-1968, 1996.

[4] D. C. Dunand and P. Müllner, "Size effects on magnetic actuation in Ni-Mn-Ga shape-memory alloys," Advanced Materials, vol. 23, no. 2, pp. 216-232, 2011.

[5] Boris K. Ponomarev, Sergey A. Ivanov, Valery D. Negrii et al., "Giant magnetic anisotropy in paramagnetic $\mathrm{Tb}_{2}\left(\mathrm{MoO}_{4}\right)_{3}$," Ferroelectrics, vol. 151, no. 1-4, pp. 103-108, 1994.

[6] A. P. Levanyuk and D. G. Sannikov, "On closed by the temperature phase transitions of the second type," JETP Letters, vol. 55, pp. 256-265, 1968.

[7] J. Petzelt and V. Dvořak, "New type of ferroelectric soft mode in gadolinium molybdate," Physica Status Solidi (b), vol. 46, pp. 413-423, 1971.

[8] J. D. Axe, B. Dorner, and G. Shirane, "Mechanism of the ferroelectric phase transformation in rare-earth molybdates," Physical Review Letters, vol. 26, no. 9, pp. 519-523, 1971.

[9] B. Dorner, J. D. Axe, and G. Shirane, "Neutron-scattering study of the ferroelectric phase transformation in $\mathrm{Tb}_{2}\left(\mathrm{MoO}_{4}\right)_{3}$," Physical Review B, vol. 6, no. 5, pp. 19501963, 1972.

[10] W. Jeitschko, "The crystal structure of ferroelectric gadolinium molybdate, $\mathrm{Gd}_{2}\left(\mathrm{MoO}_{4}\right)_{3}$," Die Naturwissenschaften, vol. 57, no. 11, p. 544, 1970.

[11] W. Jeitschko, "A comprehensive X-ray study of the ferroelectric-ferroelastic and paraelectric-paraelastic phases of $\mathrm{Gd}_{2}\left(\mathrm{MoO}_{4}\right)_{3}$," Acta Crystallographica B, vol. 28, pp. 60-76, 1972.

[12] K. Aizu, "Possible species of "Ferroelastic" crystals and of simultaneously ferroelectric and ferroelastic Crystals," Journal of the Physical Society of Japan, vol. 27, no. 2, pp. 387-396, 1969.
[13] E. Sawaguchi and L. E. Cross, "Spontaneous polarization of $\mathrm{Gd}_{2}\left(\mathrm{MoO}_{4}\right)_{3}$," Journal of Applied Physics, vol. 44, no. 6, pp. 2541-2544, 1973.

[14] H. J. Borhardt and P. E. Bierstedt, "Ferroelectric rare-earth molybdates," Journal of Applied Physics, vol. 38, no. 5, pp. 2057-2061, 1967.

[15] L. E. Cross, A. Fouskova, and S. E. Cummins, "Gadolinium molybdate, a new type of ferroelectric crystal," Physical Review Letters, vol. 21, no. 12, pp. 812-814, 1968.

[16] F. G. Ullman, B. N. Ganguly, and J. R. Zeidler, "Pyroelectric detection properties of gadolinium molybdate (gmo)," Journal of Electronic Materials, vol. 1, no. 3, pp. 425-434, 1972.

[17] F. G. Ullman, K. M. Cheung, G. A. Rakes, and B. N. Ganguly, "Re-examination of the pyroelectric current anomaly in gadolinium molybdatet," Ferroelectrics, vol. 9, pp. 63-64, 1975.

[18] R. A. Fisher, E. W. Hornung, G. E. Brodale, and W. F. Giauque, "Magnetothermodynamics of antiferromagnetic, ferroelectric $\beta-\mathrm{Gd}_{2}\left({\mathrm{M} . o \mathrm{O}_{4}}_{3}\right)_{3}$. I. Heat capacity, entropy, magnetic moment of the electrically polarized form from 0.4 to $4.2 \circ \mathrm{K}$ with Fields to $90 \mathrm{kG}$ along the c crystal axis," The Journal of Chemical Physics, vol. 56, no. 1, pp. 193-212, 1972.

[19] E. W. Hornung, G. E. Brodale, R. A. Fisher, and W. F. Giauque, "Magnetothermodynamics of antiferromagnetic, ferroelectric $\beta-\mathrm{Gd}_{2}\left(\mathrm{MoO}_{4}\right)_{3}$. II. Heat capacity, entropy, magnetic moment of the electrically polarized form from 0.4 to $4.2 \circ \mathrm{K}$ with fields to $90 \mathrm{kG}$ along the a crystal axis," The Journal of Chemical Physics, vol. 56, no. 10, pp. 5007-5018, 1972.

[20] G. E. Brodale, R. A. Fisher, E. W. Hornung, and W. F. Giauque, "Magnetothermodynamics of antiferromagnetic, ferroelectric $\beta-\mathrm{Gd}_{2}\left(\mathrm{MoO}_{4}\right)_{3}$. III. Heat capacity, entropy, magnetic moment of the electrically polarized form from 0.4 to $4.2 \circ \mathrm{K}$ with fields to $90 \mathrm{kG}$ along the $\mathrm{b}$ crystal axis," The Journal of Chemical Physics, vol. 56, no. 12, pp. 6118-6125, 1972.

[21] R. A. Fisher, E. W. Hornung, G. E. Brodale, and W. F. Glauque, "Magnetothermodynamics of antiferromagnetic, ferroelectric, ferroelastic $\beta-\mathrm{Gd}_{2}\left(\mathrm{MoO}_{4}\right)_{3}$, IV Thermodynamic temperature and other properties without heat introduction below $0.5 \circ \mathrm{K}$. Fields to $10 \mathrm{kG}$ along the $\mathrm{c}^{+}$crystal axis," The Journal of Chemical Physics, vol. 59, no. 11, pp. 5796-5809, 1973.

[22] R. A. Fisher, E. W. Hornung, G. E. Brodale, and W. F. Giauque, "Magnetothermodynamics of antiferromagnetic, polarized ferroelectric, ferroelastic $\beta-\mathrm{Gd}_{2}\left(\mathrm{MoO}_{4}\right)_{3}$. V. Thermodynamic temperature and other properties with heat introduction below $0.5 \circ \mathrm{K}$. Fields to $5 \mathrm{kG}$ along the b crystal axis," The Journal of Chemical Physics, vol. 69, no. 6, pp. 2892-2900, 1978.

[23] R. A. Fisher, E. W. Hornung, G. E. Brodale, and W. F. Giauque, "Magnetothermodynamics of ferroelectric, ferroelastic, antiferromagnetic $\beta$-terbium molybdate. I. Heat capacity, entropy, magnetic moment of the electrically polarized form from 0.4 to $4.2 \circ \mathrm{K}$ with fields to $90 \mathrm{kG}$ along the c crystal axis," The Journal of Chemical Physics, vol. 63, no. 3, pp. 1295-1308, 1975.

[24] B. K. Ponomarev, Yu. F. Popov, and D. S. Red'kin, "Magnetostriction of paramagnetic terbium molybdate in fields up to 150 kOe," JETP Letters, vol. 57, pp. 483-486, 1993.

[25] B. K. Ponomarev, S. A. Ivanov, B. S. Red'Kin, and V. N. Kurlov, "Irreversible alterations of ferroelectric domain structure in paramagnetic rare earth molybdates induced by a magnetic field," Journal of Applied Physics, vol. 75, no. 12, pp. 8004-8007, 1994. 
[26] B. K. Ponomarev, A. I. Popov, J. Van Tol et al., "Magnetism of singlets in terbium molybdate," Journal of Magnetism and Magnetic Materials, vol. 258-259, pp. 510-512, 2003.

[27] L. D. Landau and E. M. Lifshits, Kvantovaya Mekhanika, Nauka, Moscow, Russia, 1974.

[28] J. Samuel Smart, Effective Field Theories of Magnetism, IBM Watson Research Center, New York, NY, USA, 1966.

[29] H. A. Jahn and E. Teller, "Stability of polyatomic molecules in degenerate electronic states. I. Orbital degeneracy," Proceedings of the Royal Society A, vol. 161, pp. 220-235, 1937.

[30] H. A. Kramers, "Theorie generale de la rotation paramagnetique dans les cristaux," Proceedings Academy of Science, Amsterdam, vol. 33, pp. 959-972, 1930.

[31] A. K. Zvezdin, V. M. Matveev, A. A. Mukhin, and A. I. Popov, Redkozemel'nye Iony v Magnito-Uporyadochennykh Kistallakh, Nauka, Moscow, Russia, 1985.

[32] L. D. Landau and E. M. Lifshits, Electrodynamics of Continuum, Gostekhizdat, Moscow, Russia, 1957.

[33] I. E. Dzyaloshinskii, "Topological model of a heisenberg spin glass," JETP Letters, vol. 37, pp. 881-882, 1959.

[34] D. N. Astrov, "The magnetoelectric effect in antiferromagnetics,” JETP Letters, vol. 38, pp. 984-985, 1960.

[35] D. N. Astrov, "Magnetoelectric effect in chromium oxide," JETP Letters, vol. 40, pp. 1035-1041, 1961.

[36] S. L. Hou and N. Bloembergen, "Paramagnetoelectric effects in $\mathrm{NiSO}_{4} 6 \mathrm{H}_{2} \mathrm{O}$," Physical Review, vol. 138, no. 4A, pp. A1218A1226, 1965.

[37] S. A. Ivanov, V. N. Kurlov, B. K. Ponomarev, and B. S. Red'kin, "Magnetoelectric effect in terbium molibdate," JETP Letters, vol. 52, no. 7, pp. 1003-1005, 1990.

[38] B. K. Ponomarev, S. A. Ivanov, B. S. Red'kin, and V. N. Kurlov, "Magnetoelectrical effect in paramagnetic rare-earth molybdates," Physica B, vol. 177, no. 1-4, pp. 327-329, 1992.

[39] I. E. Chupis, "Effect of magnetic field on ferroelectric properties of rare-earthe molobdates," Fizika Nizkikh Temperatur, vol. 21, no. 9, pp. 941-4695, 1995.

[40] B. K. Ponomarev, S. A. Ivanov, B. S. Red'kin, and V. N. Kurlov, "Effect of a magnetic field on the ferroelectric domain structure of $\mathrm{TbGd}\left(\mathrm{MoO}_{4}\right)_{3}$," JETP Letters, vol. 55, pp. 353361, 1992.

[41] B. K. Ponomarev, V. D. Negrii, B. S. Red'kin, and Yu F. Popov, "Magneto-electro-elastic effects in some rare earth molybdates and related properties," Journal of Physics D: Applied Physics, vol. 27, no. 10, pp. 1995-2001, 1994.

[42] H. Wiegelmann, B. K. Ponomarev, J. van Tol, A. G. M. Jansen, P. Wyder, and B. S. Red'kin, "Magnetoelectric properties of ferroelectric rare earth molybdates," Ferroelectrics, vol. 183, pp. 195-204, 1996.

[43] B. K. Ponomarev, A. I. Popov, E. Steep et al., Fizika Tverdogo Tela, vol. 47, no. 7, p. 1326, 2005.

[44] N. F. Vedernikov, A. K Zvezdin, R. Z. Levitin, and A. I. Popov, "Magnetic linear birefringence of rare-earth garnets," JETP Letters, vol. 93, p. 2161, 1987, Soviet Physics-JETP, vol. 66, no. 6, p. 1233, 1987.

[45] N. S. Akulov, Ferromagnetics, Gostekhizdat, Moscow, Russia, 1939.

[46] E. Callen and H. B. Callen, "Magnetostriction, forced magnetostriction, and anomalous thermal expansion in ferromagnets," Physical Review, vol. 139, no. 2A, pp. A455-A471, 1965.

[47] B. K. Ponomarev, B. S. Red'kin, H. Wiegelmann, A. G. M. Jansen, P. Wyder, and J. van Tol., "Magnetoelectric effect in orthorhombic $\mathrm{Gd}_{2}\left(\mathrm{MoO}_{4}\right)_{3}$," Ferroelectric letters, vol. 18, no. 3-4, pp. 133-140, 1994.
[48] B. K. Ponomarev, E. Steep, H. Wiegelmann, A. G. M. Jansen, P. Wyder, and B .S. Red'kin, "Anisotropy of the magnetoelectric effect in $\beta^{\prime}-\mathrm{Gd}_{2}\left(\mathrm{MoO}_{4}\right)_{3}$," Physics of the Solid State, vol. 42, no. 4, pp. 716-738, 2000.

[49] H. B. Callen and E. Callen, "The present status of the temperature dependence of magnetocrystalline anisotropy, and the $1(1+1) 2$ power law," Journal of Physics and Chemistry of Solids, vol. 27, no. 8, pp. 1271-1285, 1966.

[50] B. K. Ponomarev, B. S. Red'kin, E. Steep, H. Wiegelmann, A. G. M. Jansen, and P. Wyder, "Magnetoelectric effect in samarium molybdate," Physics of the Solid State, vol. 44, no. 1, pp. 145148, 2002.

[51] K. P. Belov, M. A. Belyanchokova, R. Z. Levitin, and S. A. Nikitin, Rare-Earth Ferromagnets and Antiferromagnets, Nauka, Moscow, Russia, 1965.

[52] B. K. Ponomarev, J. Zeman, G. Martinez et al., "The anisotropy of the intensity of the optical absorption in $\beta^{\prime}-\mathrm{Tb}_{2}\left(\mathrm{MoO}_{4}\right)_{3}$," Ferroelectrics, vol. 204, pp. 279-288, 1997.

[53] B. K. Ponomarev, A. I. Popov, B. S. Red'kin et al., "The longitudinal zeeman effect in terbium molybdate," Journal of Magnetism and Magnetic Materials, vol. 300, no. 1, pp. e422e425, 2006.

[54] B. K. Ponomarev, I. A. Kornev, V. D. Negrii, G. M. Vizdrik, and B. S. Red'kin, "Anomalously high photovoltages in terbium molybdate," Physics of the Solid State, vol. 40, pp. 661-663, 1998.

[55] B. K. Ponomarev, V. D. Negrii, and B. S. Red'Kin, "Two types of photo-induced voltages in terbium molybdate," Ferroelectrics, vol. 280, pp. 119-130, 2002.

[56] A. A. Grekov, M. A. Malitskaia, V. D. Spitsina, and B. M. Fridkin, "Photoelectric effects in A5B6C7-type ferroelectricssemiconductors with low-temperature phase transitions," Kristallografiya, vol. 15, pp. 500-509, 1970.

[57] A. M. Glass, D. von der Linde, and T. J. Negran, "High-voltage bulk photovoltaic effect and the photorefractive process in $\mathrm{LiNbO}_{3}$," Applied Physics Letters, vol. 25, no. 4, pp. 233-235, 1974.

[58] V. M. Fridkin, Fotosegnetoelektriki, Nauka, Moscow, Russia, 1979.

[59] A. G. Chynoweth, "Surface space-charge layers in barium titanate," Physical Review, vol. 102, no. 3, pp. 705-714, 1956.

[60] P. V. Ionov, "Photosensitivity of ferroelectric niobates," Fizika Tverdogo Tela, vol. 15, no. 9, pp. 2827-2878, 1973.

[61] P. V. Ionov, V. V. Voronov, and V. T. Gabrielyan, "Detection of photoinduced change of refraction in ferroelectric lead germanate," Fizika Tverdogo Tela, vol. 17, no. 4, pp. 11441146, 1975.

[62] V. M. Fridkin, B. N. Popov, and K. A. Verkhovskaya, "Photovoltaic and photorefractive effects in ferroelectrics of kdpgroup," Fizika Tverdogo Tela, vol. 20, no. 4, pp. 1263-1265, 1978.

[63] B. K. Ponomarev and B. S. Red'kin, "Fluctuations of the piezoacoustic impedance in gadolinium molybdate near the curie temeprature," Fiz. Tverd. Tela, vol. 49, pp. 1260-1264, 2007.

[64] "IRE standards on piezoelectric crystals," Proceedings of the IR, vol. 49, no. 7, pp. 1161-1169, 1961.

[65] K. S. van Dyke, "The piezo-electric resonator and its equivalent network," Proceedings of the IRE, no. 16, pp. 742-764, 1928.

[66] L. Bergmann, Der Ultraschall und seine Anwendung in Wissenschaft und Technik, Zürich, Switzerland, 1954.

[67] I. A. Yakovlev and T. S. Velichkina, "Two new phenomena at phase transitions of second type," Uspekhi Fizicheskikh Nauk, vol. 63, no. 10, pp. 411-433, 1957. 

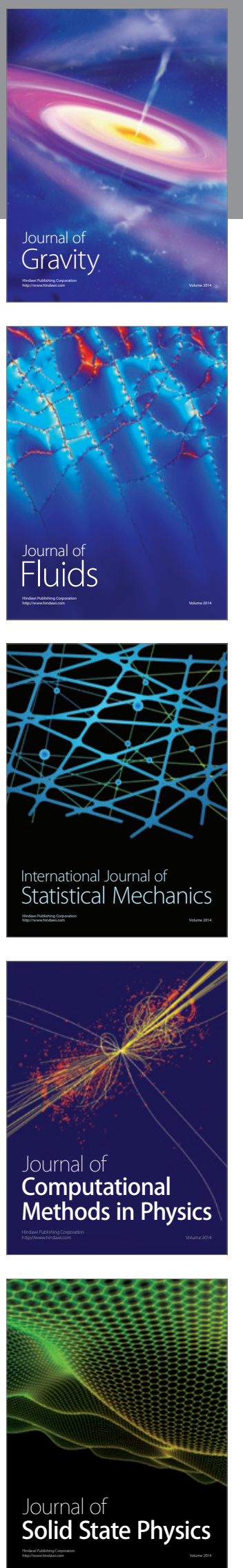

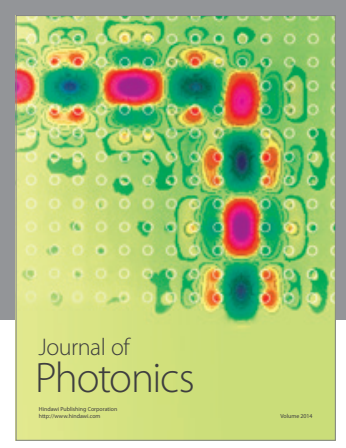

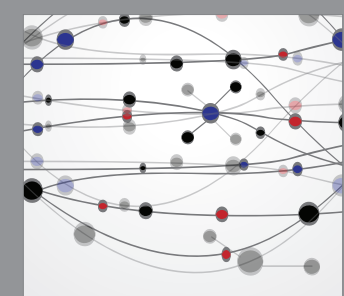

The Scientific World Journal
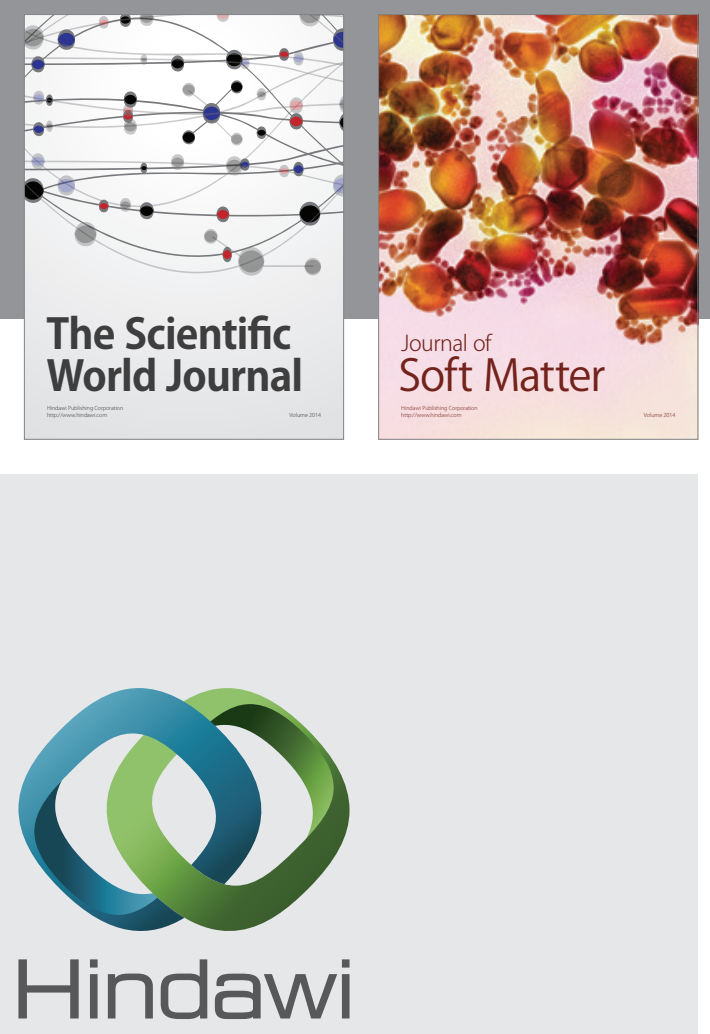

Submit your manuscripts at

http://www.hindawi.com
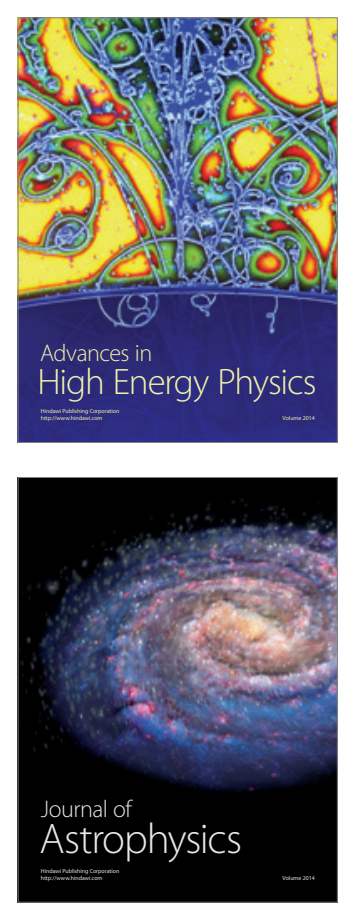
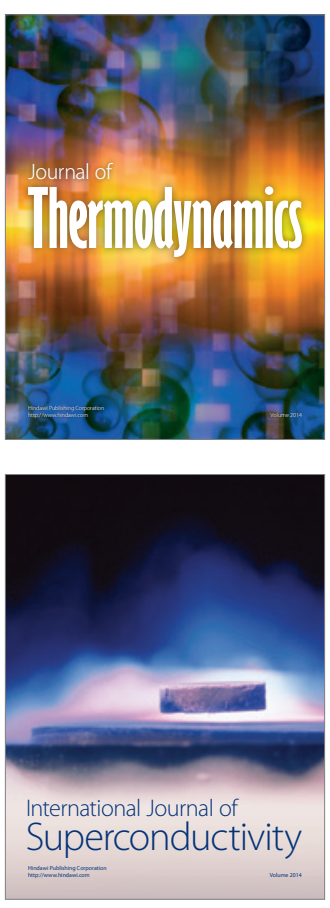
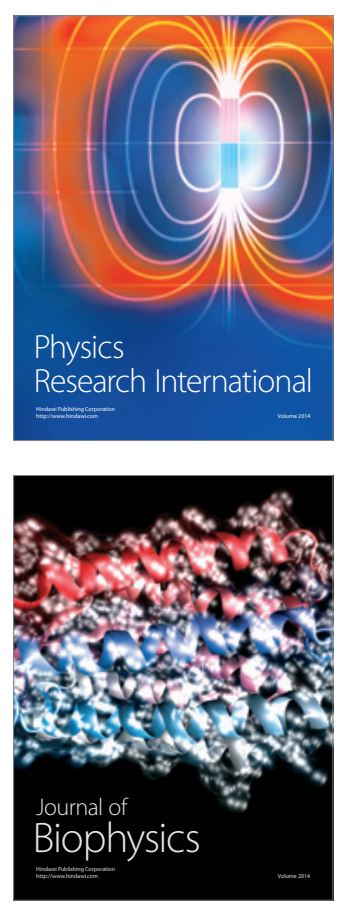
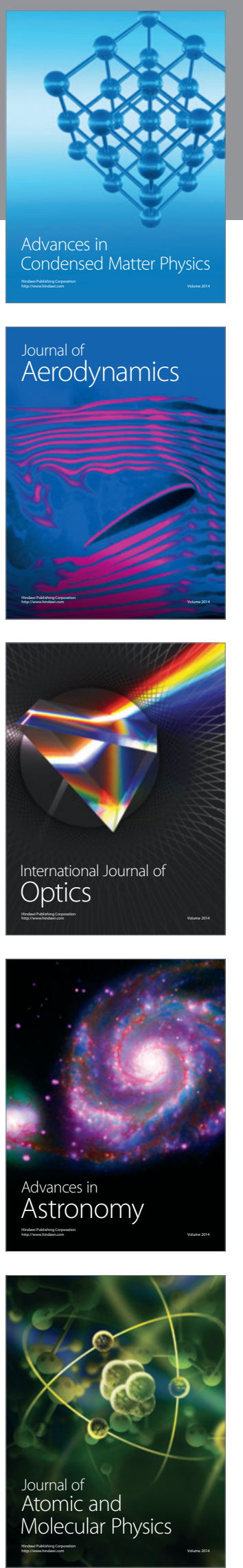